\title{
Hadronic decays of a light Higgs-like scalar
}

\author{
Alexander Monin, ${ }^{1, *}$ Alexey Boyarsky, ${ }^{2}$ and Oleg Ruchayskiy ${ }^{3}$ \\ ${ }^{1}$ Department of Theoretical Physics, University of Geneva 24 quai Ernest-Ansermet, 1211 Geneva \\ ${ }^{2}$ Lorentz, Institute, Leiden University, Niels Bohrweg 2, Leiden, NL-2333 CA, Netherlands \\ ${ }^{3}$ Discovery Center, Niels Bohr Institute, Copenhagen University, \\ Blegdamsvej 17, DK-2100 Copenhagen, Denmark
}

(Received 27 August 2018; published 10 January 2019)

\begin{abstract}
We revisit the question of hadronic decays of a giga-electron-volt-mass Higgs-like scalar. A number of extensions of the Standard Model predict the Higgs sector with additional light scalars. Currently operating and planned Intensity Frontier experiments will probe for the existence of such particles, while theoretical computations are plagued by uncertainties. The goal of this paper is to bring the results in a consolidated form that can be readily used by experimental groups. To this end, we provide a physically motivated fitting ansatz for the decay width that reproduces the previous nonperturbative numerical analysis. We describe systematic uncertainties of the nonperturbative method and provide explicit examples of the influence of extra resonances above $1.4 \mathrm{GeV}$ onto the total decay width.
\end{abstract}

DOI: 10.1103/PhysRevD.99.015019

\section{MOTIVATION}

The Standard Model of particle physics provides a closed and self-consistent description of known elementary particles interacting via strong, weak, and electromagnetic forces. The Standard Model coupled with general relativity has also been very successful in describing the evolution of the Universe as a whole. However, the impressive success of the Standard Model at accelerator and cosmic frontiers has also revealed with certainty that the Standard Model fails to explain a number of observed phenomena in particle physics, astrophysics, and cosmology. These major unsolved challenges are commonly known as "beyond the Standard Model" (BSM) problems. They include neutrino masses and oscillations, dark matter, baryon asymmetry of the Universe, etc.

A range of possible scenarios capable of resolving the BSM puzzles is extremely wide. At the one end, there are models such as the neutrino minimal Standard Model $(\nu \mathrm{MSM})[1,2]$ that postulate only three extra particles lighter than electroweak scale, providing resolutions of major BSM puzzles and leading to a Standard Model-like quantum field theory up to very high scales [3-5]. At the other end, there are models in which one is completely agnostic about the structure of the hidden ("dark") sectors

\footnotetext{
*alexander.monin@unige.ch
}

Published by the American Physical Society under the terms of the Creative Commons Attribution 4.0 International license. Further distribution of this work must maintain attribution to the author(s) and the published article's title, journal citation, and DOI. Funded by SCOAP. and explores portals - mediator particles that both couple to states in the "hidden sectors" and interact with the Standard Model. Such portals can be renormalizable (mass dimension $\leq 4$ ) or be realized as higher-dimensional operators suppressed by the dimensionful couplings $\Lambda^{-n}$, with $\Lambda$ being the new energy scale of the hidden sector. Mediator couplings to the Standard Model sector can be sufficiently small to allow for the portal particles to be (much) lighter than the electroweak scale. Such models can be explored with Intensity (rather than Energy) frontier experiments.

In this paper, we focus on scalar (or "Higgs") portal [6] - gauge singlet scalar $S$ interacting with the Higgs doublet $H$ via the $S H^{\dagger} H$ term. Such particles "inherit" their interactions from the Higgs boson (albeit suppressed by a small dimensionless parameter $\theta$ ). New generation of Intensity Frontier experiments, such as NA62 [7-9], SHiP [10,11], MATHUSLA [12,13], FASER [14]), CODEX-b [15], and SeaQuest [16] will probe for the existence of such scalars with the masses $\sim \mathrm{GeV}$. The lifetime of such scalars is dominated by the decay into light mesons ( $S \rightarrow \pi \pi, S \rightarrow \bar{K} K$, etc.) The question of computation of the decay width of such particles was studied in the 1980s [17-23] in the context of hadronic decays of the light Higgs boson. Based on the data for $\psi^{\prime} \rightarrow \psi \pi \pi$ and $\Upsilon^{\prime} \rightarrow \Upsilon^{\prime} \pi \pi$ decays, Ref. [18] argued in favor of extrapolating the results obtained with the help of chiral perturbation theory (ChPT) up to $1.5 \mathrm{GeV}$. At the same time, the nonperturbative analysis of Ref. [23] produced results differing from Ref. [18] by as much as an order of magnitude.

This discrepancy, crucial for the new generation of experiments, warrants the current work. We critically 
review existing methods of computation of the scalar's hadronic width and assess the uncertainties. We mainly reconfirm findings of Ref. [23] but provide a way to assess its uncertainties and speculate up to what scales the nonperturbative approach should be used (read: trusted).

The paper is organized as follows. In Sec. II, we discuss briefly the properties of the scalar portal and define the form factors through which the hadronic decay width $\Gamma_{\pi \pi}$ is expressed. We review computation of the hadronic decay in the chiral perturbation theory, reproducing the results of Ref. [18] in Sec. III. The unitarity arguments that allow for nonperturbative treatment of the relevant form factors are summarized in Sec. IV. The review of dispersive methods is given in Sec. V. Section VI summarizes our results and compares with previous works. Section VII provides the error estimate and domain of validity. We conclude in Sec. VIII and provide supplementary material in the Appendices.

\section{SETUP}

We start by laying down the ground rules for computing the desired decay rate. We consider a scalar field $S$ weakly coupled to the Standard Model Higgs field $H$; see Ref. [10] for details. For masses below $1 \mathrm{GeV}$, the relevant UV couplings of the scalar are only those to quarks and leptons

$$
\mathcal{L}_{\text {int }}=-\frac{S}{v_{S}} \sum_{q} m_{q} \bar{q} q-\frac{S}{v_{S}} \sum_{\ell} m_{\ell} \bar{\ell} \ell,
$$

since the only interesting decay channels are $\pi \pi, \mu^{+} \mu^{-}$, and possibly $\bar{K} K$. In Eq. (1), $v_{S} \equiv v \cot \theta$, where $v=246 \mathrm{GeV}$ is the Higgs vacuum expectation value (vev) and $\theta \ll 1$ parametrizes the interaction of the scalar $S$ with the Standard Model particles.

From the Lagrangian (1), the decay rate $S \rightarrow \mu^{+} \mu^{-}$can be immediately found:

$$
\Gamma_{\mu^{+} \mu^{-}}=\frac{1}{8 \pi} \frac{m_{\mu}^{2} m_{S}}{v_{S}^{2}}\left(1-\frac{4 m_{\mu}^{2}}{m_{S}^{2}}\right)^{3 / 2} .
$$

Computing the width due to hadronic decays is somewhat more involved. The difficulty stems from the strong coupling of QCD in the regime of interest. Therefore, considering only the tree-level process - which in the case of the leptonic decay leads to (2) - is not enough. Quarks and gluons are not adequate degrees of freedom for describing the low-energy physics. Instead, to compute hadronic decay rates, matrix elements of the Lagrangian (1) between low-energy hadronic states should be computed directly. For instance, in the case of $S \rightarrow \pi^{a} \pi^{b}$, where $a$ and $b$ are isospin indices, the amplitude is defined as

$$
\begin{aligned}
\mathcal{A}_{\pi}\left(m_{S}^{2}\right) \delta^{a b} & \equiv\left\langle\pi^{a}\left(p_{1}\right) \pi^{b}\left(p_{2}\right)\left|i \mathcal{L}_{\mathrm{int}}\right| S\right\rangle \\
& =-\frac{i}{v_{S}}\left\langle\pi^{a}\left(p_{1}\right) \pi^{b}\left(p_{2}\right)\left|\sum_{q} m_{q} \bar{q} q\right| 0\right\rangle
\end{aligned}
$$

Integrating it over the phase space gives the decay width

$$
\Gamma_{\pi \pi}=\frac{3}{32 \pi} \frac{\left|\mathcal{A}_{\pi}\left(m_{S}^{2}\right)\right|^{2}}{m_{S}} \sqrt{1-\frac{4 m_{\pi}^{2}}{m_{S}^{2}}}
$$

where we summed over all species and took into account that the particles in the final state are identical.

The sum in (3) contains contributions from light $(u, d, s)$ and heavy $(c, b, t)$ quarks. The latter can be expressed in terms of the former and the energy-momentum tensor by using a clever trick based on the knowledge of the trace anomaly and the renormalization group invariance of the energy-momentum tensor (for more details, see Refs. [18,24,25] and [20,21,26]). It uses two different representations of the energy-momentum tensor at energies immediately above and below the $c$-quark mass. On the one hand, using the UV description (all quarks), the trace of the energy-momentum tensor, due to the anomaly, is given by

$\theta_{\mu}^{\mu}=\frac{\beta\left(\alpha_{s}\right)}{4 \alpha_{s}} G^{2}+\sum_{\text {all }} m_{q} \bar{q} q, \quad \alpha_{s}=\frac{g_{s}^{2}}{4 \pi}, \quad G^{2}=G_{\mu \nu}^{a} G_{\mu \nu}^{a}$,

with the one-loop beta function for the strong coupling $\alpha_{s}$ defined as

$$
\beta\left(\alpha_{s}\right)=-\frac{b \alpha_{s}^{2}}{2 \pi}, \quad b=9-\frac{2}{3} N_{h},
$$

where $N_{h}$ is the number of heavy quarks (in our case 3 ). On the other hand, from the IR perspective (after integrating out heavy quarks), the energy-momentum tensor becomes

$$
\theta_{\mu}^{\mu}=\frac{\bar{\beta}\left(\alpha_{s}\right)}{4 \alpha_{s}} G^{2}+\sum_{\text {light }} m_{q} \bar{q} q+O\left(1 / m_{c}^{2}\right)
$$

where the reduced beta function corresponds to only light quarks $(u, d, s)$,

$$
\bar{\beta}\left(\alpha_{s}\right)=-\frac{9 \alpha_{s}^{2}}{2 \pi} .
$$

As a result, one concludes that

$$
\sum_{\text {heavy }} m_{q} \bar{q} q=-\frac{2}{3} N_{h} \frac{\alpha_{s}}{8 \pi} G^{2}=\frac{2}{27} N_{h}\left(\theta_{\mu}^{\mu}-\sum_{\text {light }} m_{q} \bar{q} q\right),
$$

and the interaction Lagrangian can therefore be rewritten as 


$$
\mathcal{L}_{\text {int }}=-\frac{S}{v_{S}}\left[\frac{2}{27} N_{h} \theta_{\mu}^{\mu}+\left(1-\frac{2}{27} N_{h}\right) \sum_{\text {light }} m_{q} \bar{q} q\right],
$$

which immediately leads to the expression for the amplitude (3) in the leading order in $\alpha_{s}$,

$$
\begin{aligned}
A_{\pi}\left(m_{S}^{2}\right)= & \frac{i}{v_{S}}\left\{\frac{2}{27} N_{h} \theta_{\pi}\left(m_{S}^{2}\right)\right. \\
& \left.+\left[1-\frac{2}{27} N_{h}\right]\left[\Gamma_{\pi}\left(m_{S}^{2}\right)+\Delta_{\pi}\left(m_{S}^{2}\right)\right]\right\},
\end{aligned}
$$

where the following notations for the form factors were introduced:

$$
\begin{aligned}
& \Gamma_{\pi}(s) \delta^{a b}=\left\langle\pi^{a} \pi^{b}\left|m_{u} \bar{u} u+m_{d} \bar{d} d\right| 0\right\rangle, \\
& \Delta_{\pi}(s) \delta^{a b}=\left\langle\pi^{a} \pi^{b}\left|m_{s} \bar{s} s\right| 0\right\rangle, \\
& \theta_{\pi}(s) \delta^{a b}=\left\langle\pi^{a} \pi^{b}\left|\theta_{\mu}^{\mu}\right| 0\right\rangle .
\end{aligned}
$$

The problem of computing the width (4) thus boils down to computing these form factors. In the next sections, we present several approximations when it can be done using different techniques such as the ChPT and unitarity. It is also important to note that the expression (11) does not capture effects suppressed by heavy quark masses $1 / m_{c}^{2}$.

\section{ChPT}

For very small energies, the form factors (12a)-(12c) can be easily computed using the chiral perturbation theory $[18,27]$. We will not go to great lengths to introduce the ChPT and refer the reader to numerous sources (for instance, Refs. [28-30]). Instead, we give just the key results allowing us to demonstrate how the computation is done.

The low-energy dynamics of QCD can be described [in the case of the $S U(2) \times S U(2)$ chiral symmetry] by introducing an $S U(2)$ matrix,

$$
\Sigma=e^{i \sigma_{a} \pi_{a} / f_{\pi}}
$$

parametrized by pion fields $\pi_{a}$, with $f_{\pi}=93 \mathrm{MeV}$ being the pion decay constant. The action of the chiral symmetry group on the space of these matrices is realized by the right and left multiplications,

$$
\Sigma^{\prime}=U_{L} \Sigma U_{R}^{\dagger} .
$$

In building the Lagrangian, one has to make sure that the symmetry (14) is preserved. It is straightforward to show that the leading (derivative expansion) order Lagrangian is given by

$$
L=\frac{f_{\pi}^{2}}{4} \operatorname{Tr} \partial_{\mu} \Sigma \partial_{\mu} \Sigma^{\dagger}+\frac{B f_{\pi}^{2}}{2} \operatorname{Tr}\left(M^{\dagger} \Sigma+\Sigma^{\dagger} M\right),
$$

where $M$ is the quark mass matrix

$$
M=\left(\begin{array}{cc}
m_{u} & 0 \\
0 & m_{d}
\end{array}\right)
$$

and $B$ is a constant. Expanding the Lagrangian up to quadratic order shows that the pion mass is given by $m_{\pi}^{2}=B\left(m_{u}+m_{d}\right)$. At this order, the trace of the energymomentum tensor is given by

$$
\theta_{\mu}^{\mu}=2 m_{\pi}^{2} \pi^{2}-(\partial \pi)^{2} .
$$

Therefore, the corresponding form factor (12c) becomes

$$
\theta_{\pi}(s)=s+2 m_{\pi}^{2} .
$$

The other two form factors (12a) and (12b) can be computed in a similar way:

$$
\Gamma_{\pi}(s)=m_{\pi}^{2}, \quad \Delta_{\pi}(s)=0 .
$$

As a result, the amplitude (11) becomes [18]

$$
\mathcal{A}_{\pi}=\frac{2}{9} \frac{i}{v_{S}}\left(m_{S}^{2}+\frac{11}{2} m_{\pi}^{2}\right),
$$

which — upon using (4) —leads to the following expression for the $S \rightarrow \pi \pi$ decay rate:

$$
\Gamma_{\pi \pi}=\frac{1}{216 \pi} \sqrt{1-\frac{4 m_{\pi}^{2}}{m_{S}^{2}}} \frac{1}{m_{S} v_{S}^{2}}\left(m_{S}^{2}+\frac{11}{2} m_{\pi}^{2}\right)^{2} .
$$

By construction, the ChPT is only reliable for sufficiently small energies. It is thus clear that the result (20) is valid at energies low compared to QCD scale, i.e., $\Lambda_{\mathrm{QCD}} \equiv 4 \pi f_{\pi} \approx 1 \mathrm{GeV}$. At the same time, we are interested in finding the decay rate for scalars with masses comparable to $1 \mathrm{GeV}$. To do that, it is not enough to compute the next-to-leading-order correction in ChPT. In the next section, we will present a nonperturbative approach based on dispersion relations.

\section{BEYOND ChPT AND UNITARITY}

In the previous section, we showed how the decay rate of a light scalar into pions can be computed using the power of the effective field theory. ChPT corrections to the leading-order result are suppressed by powers of $s / \Lambda_{\mathrm{QCD}}^{2}$. Therefore, perturbative computations become unreliable at energies close to the cutoff. However, the precise point where corrections become comparable with the leadingorder computation depends on the specifics of an observable. 
There are indications that for the form factor $\Gamma_{\pi}(s)$ it happens for energies $s$ much smaller ${ }^{1}$ than $\Lambda_{\mathrm{QCD}}^{2}$.

Using the definition of the quadratic scalar radius of the pion

$$
\left\langle r^{2}\right\rangle_{S, \pi}=\left.6 \frac{\partial \log \Gamma_{\pi}(s)}{\partial s}\right|_{s=0},
$$

the form factor $\Gamma_{\pi}(s)$ around $s=0$ can be written as

$$
\Gamma_{\pi}(s)=\Gamma_{\pi}(0)\left(1+\frac{1}{6} s\left\langle r^{2}\right\rangle_{S, \pi}+\ldots\right),
$$

where $\Gamma_{\pi}(0)$ is given by Eq. (19). The quadratic scalar radius of the pion was first computed in Ref. [32] using ChPT at one loop, with the result $\left\langle r^{2}\right\rangle_{S, \pi}=0.55 \pm 0.15 \mathrm{fm}^{2}$. The method of Ref. [23] (to be discussed shortly) produced $\left\langle r^{2}\right\rangle_{S, \pi}=0.600 \pm 0.052 \mathrm{fm}^{2}$. Later, using a better input for the pi-pi phases, the analysis was repeated in Ref. [33], producing

$$
\left\langle r^{2}\right\rangle_{S, \pi}=0.61 \pm 0.04 \mathrm{fm}^{2} .
$$

Recently, this computation was corroborated by lattice computations in Ref. [34]. ${ }^{2}$ Such a value of the quadratic scalar radius implies that $\mathrm{ChPT}$ cannot be trusted when $\frac{1}{6} s\left\langle r^{2}\right\rangle_{S, \pi} \sim 1$ or, equivalently, at $\sqrt{s} \sim 600-700 \mathrm{MeV}$ (see also Refs. [20,21]). Therefore, for masses of a scalar $m_{S} \lesssim 1 \mathrm{GeV}$, a nonperturbative approach should be used.

\section{A. Analyticity and unitarity}

Such a method by definition should use only the most general constraints on form factors without alluding-if possible - to any specific perturbative computation. The first constraint comes from analyticity. It can be proven (see Refs. $[35,36])$ that form factors are analytic functions in the complex plane of the variable $s$ with the cut $s>4 m_{\pi}^{2}$, and it can be established (using the high-energy behavior of QCD [37]) that their behavior at infinity should be $\sim 1 / s$.

The second constraint is due to unitarity. To discuss it, we have to introduce the notion of the scattering matrix for $\mathrm{s}$ waves with isospin zero. For two-to-two $\left(\pi^{a} \pi^{b} \rightarrow \pi^{c} \pi^{d}\right)$ scattering, the $S$ matrix, defined as

$$
S_{a b c d}(s, t, u)={ }_{\text {out }}\left\langle\pi_{c}\left(p_{3}\right) \pi_{d}\left(p_{4}\right) \mid \pi_{a}\left(p_{1}\right) \pi_{b}\left(p_{2}\right)\right\rangle_{\text {in }},
$$

depends on all Mandelstam variables $(s, t$, and $u$ ) and has an arbitrary tensor structure in the space of isospin indices $a, \ldots, d$. However, it can be expanded in partial waves with

\footnotetext{
${ }^{1}$ It is known that final-state interaction effects can be rather strong [31].

${ }^{2}$ It is precisely the result of Ref. [33] and lattice computations that will be used in the following sections to fix unknown coefficients in several form factors.
}

fixed angular momentum $J$ and isospin $I$. We are interested in scalar (isoscalar) form factors; therefore, we consider only s-wave isospin-0 $(J=I=0)$ scattering, by projecting (25) on the corresponding subspace (see Chap. 19 in Ref. [28]). It is this component that we refer to as an $S$ matrix in what follows. For energies below the inelastic threshold $\left(4 m_{\pi}\right)$, the $S$ matrix is completely determined by the pion phase shift

$$
S(s)=e^{2 i \delta_{\pi}(s)}, \quad 4 m_{\pi}^{2}<s<16 m_{\pi}^{2} .
$$

As energy grows, channels $2 \pi \rightarrow 4 \pi, 2 \pi \rightarrow 6 \pi$, and then $2 \pi \rightarrow \bar{K} K$ open up. Correspondingly, the $S$ matrix can be represented by a finite-dimensional matrix, $S_{i j}$, with $i$ and $j$ running in the space of channels [38]. It is observed experimentally that the mixing with multiparticle (four and more) states for energies below $\Lambda_{\text {dat }}=1.4-1.6 \mathrm{GeV}$ is small $[39,40]$. Therefore, in this region, there are effectively only two relevant channels $(\pi \pi \rightarrow \pi \pi, \pi \pi \rightarrow \bar{K} K)$.

In the case of two channels - the generalization to an arbitrary number of channels seems straightforwardunitarity constraints for form factors, similar to the optical theorem (see, e.g., the textbook [41], Sec. 6-3-4) for amplitudes, can be derived in the following way. We define

$$
\begin{aligned}
& \phi_{1}(s) \delta^{a b} \equiv\left\langle\pi^{a} \pi^{b}|X| 0\right\rangle, \\
& \phi_{2}(s) \delta^{\alpha \beta} \equiv \frac{2}{\sqrt{3}}\left\langle K^{\alpha} K^{\beta}|X| 0\right\rangle,
\end{aligned}
$$

where $X$ is any of the operators appearing in ((12a)-(12c). The relative factor $2 / \sqrt{3}$ is due to the normalization of the isospin-0 eigenstates

$$
|\pi \pi\rangle=\frac{1}{\sqrt{3}} \sum_{a=1}^{3}\left|\pi^{a} \pi^{a}\right\rangle, \quad \text { and } \quad|K K\rangle=\frac{1}{2} \sum_{a=1}^{4}\left|K^{\alpha} K^{\alpha}\right\rangle .
$$

Also, we introduce the $T$ matrix via

$S_{i j}(s) \equiv \delta_{i j}+2 i T_{i j}(s) \sqrt{\sigma_{i}(s) \sigma_{j}(s)} \Theta\left(s-4 m_{i}^{2}\right) \Theta\left(s-4 m_{j}^{2}\right)$,

where $\Theta(s)$ is the Heaviside theta function (representing the opening of the corresponding channel), and the factors $\sigma_{i}(s)$

$$
\sigma_{i}(s) \equiv \sqrt{1-\frac{4 m_{i}^{2}}{s}}
$$

are responsible for the phase space volume. One can be straightforwardly convinced that the unitarity of the $S$ matrix [which is represented schematically in Fig. 1(a)] translates into the following constraint on $T$ : 


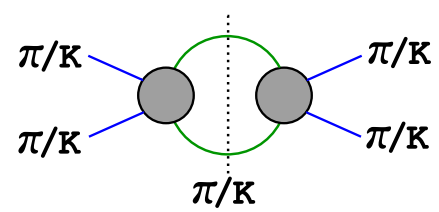

(a) Schematic representation for the unitarity condition: S-matrix

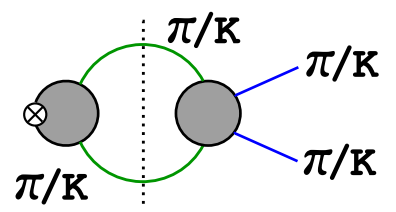

(b) Specialization of unitarity condition for the case of form-factors $\phi_{i}(s)$ in the two-channel approximation

FIG. 1. Unitarity conditions for form factors and $S$ matrix.

$$
\begin{aligned}
\operatorname{Im} T_{k i}(s) & =\sum_{j=1}^{2} T_{i j}^{*}(s) T_{k j}(s) \sigma_{j}(s) \Theta\left(s-4 m_{j}^{2}\right), \\
4 m_{\pi}^{2} & <s<\Lambda_{\mathrm{dat}}^{2} .
\end{aligned}
$$

In a complete analogy, the following relation between the imaginary part of the form factors (27) and the scattering matrix [Fig. 1(b)] can be obtained,

$$
\begin{aligned}
& \operatorname{Im} \phi_{i}(s)=\sum_{j=1}^{2} T_{i j}^{*}(s) \phi_{j}(s) \sigma_{j}(s) \Theta\left(s-4 m_{j}^{2}\right), \\
& 4 m_{\pi}^{2}<s<\Lambda_{\mathrm{dat}}^{2},
\end{aligned}
$$

which we rewrite in an equivalent form,

$$
\phi_{i}(s)=\sum_{j=1}^{2} G_{i j}(s) \phi_{j}^{*}(s), \quad 4 m_{\pi}^{2}<s<\Lambda_{\mathrm{dat}}^{2},
$$

upon defining

$G_{i j} \equiv \delta_{i j}+2 i T_{i j}(s) \sigma_{j}(s) \Theta\left(s-4 m_{j}^{2}\right)$ for $i, j=1,2$.

For energies above $\Lambda_{\text {dat }}$, other channels come into play, correspondingly modifying the constraints [in particular, extending the sum in (31)-(33) to $j>2$ ].

In deriving the system of Equations (32), one does not rely on perturbative computations. Therefore, the analogue of the system with all channels taken into account together with analyticity encapsulate all the necessary nonperturbative information about the form factors. In the next section, we will discuss several cases when the solution of (32) can be found.

\section{REVIEW OF DIFFERENT METHODS AND THEIR RESULTS}

The system (32) is similar (though not exactly the same) to a more general Hilbert problem of finding a holomorphic vector function $\phi_{i}(s)$ of finite degree at infinity with a specific boundary condition (discontinuity) on the cut(s). It is proven (see Ref. [42] and the book [43]) $)^{3}$ that the number

\footnotetext{
${ }^{3}$ For a more recent presentation, see Refs. [44-48].
}

of linearly independent canonical (having no zeros at finite points) solutions, denoted by $\Omega_{i}^{(1)}(s), \Omega_{i}^{(2)}(s), \ldots$, coincides with the number of channels. The general solution can be represented as a linear combination of the canonical ones,

$$
\phi(s)=P_{1}(s) \Omega^{(1)}+P_{2}(s) \Omega^{(2)}+\ldots,
$$

with $P_{i}(s)$ being polynomials. Moreover, the degree at infinity of the function

$$
D(s)=\operatorname{det}_{i j} \Omega_{i}^{(j)}(s)
$$

is completely fixed by the asymptotic behavior of the corresponding $S$ matrix [see a comment after Eq. (44)]. However, only in a limited number of cases (specific form of discontinuity, or in our case of the $S$ matrix), the solution can be found. Below, we describe several such cases, first showing how the solution can be derived in general and then using the real data for each of them.

\section{A. One-channel solution}

The first example in which the solution can be found explicitly is a hypothetical case of only one channel. As was discussed above, the $S$ matrix in this case is completely specified by just one phase, and the system of equations (32) reduces to only one equation:

$$
\phi(s)=e^{2 i \delta(s)} \phi^{*}(s), \quad s \in \mathbb{R} .
$$

Since the scattering phase $\delta(s)=0$ below the threshold $4 m_{\pi}^{2}$, the function $\phi(s)$ satisfies the following condition in the whole complex plane with the cut

$$
\phi\left(s^{*}\right)=\phi^{*}(s) .
$$

Assuming that the function $\phi(s)$ has zeros at $s_{1}, s_{2}, \ldots, s_{N}$, it is easy to show that the function

$$
\Omega(s)=\frac{\phi(s)}{\prod_{i=1}^{N}\left(s-s_{i}\right)}
$$

has obviously no zeros and satisfies the same equation (37). ${ }^{4}$ As a result,

$$
\operatorname{Im} \log \Omega(s)=\delta(s), \quad s \in \mathbb{R},
$$

and the normalized $\Omega(0)=1$ canonical solution, which in this case is called the Omnès factor, can be easily reconstructed by integrating $\log \Omega(s)$ with one subtraction along the contour in Fig. 2,

\footnotetext{
${ }^{4}$ Complex zeros come in pair with their complex conjugates due to (38); thus, the product in (39) is real.
} 


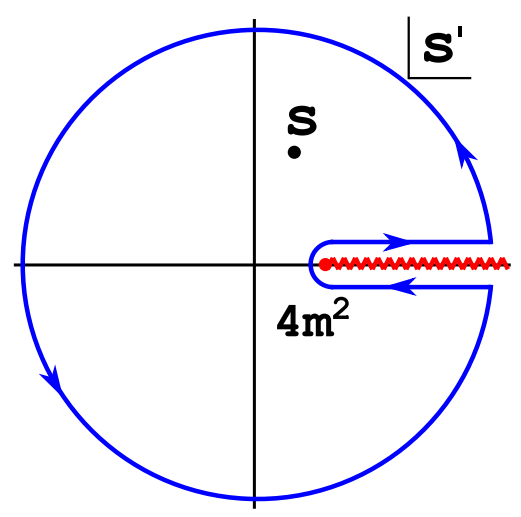

FIG. 2. Integration contour.

$$
\frac{\log \Omega(s)}{s}=\frac{1}{\pi} \int_{4 m^{2}}^{\infty} \frac{d s^{\prime}}{s^{\prime}} \frac{\delta\left(s^{\prime}\right)}{s^{\prime}-s-i \varepsilon},
$$

or equivalently

$$
\Omega(s)=\exp \left[\frac{s}{\pi} \int_{4 m^{2}}^{\infty} \frac{d s^{\prime}}{s^{\prime}} \frac{\delta\left(s^{\prime}\right)}{s^{\prime}-s-i \varepsilon}\right] .
$$

It follows from (39) that the most general solution (which can have zeros) is given by the product of the Omnès factor and a polynomial $P_{\phi}(s)$ with real coefficients, the zeros of which are fixed by that of $\phi(s)$,

$$
\phi(s)=P_{\phi}(s) \Omega(s)
$$

It is straightforward to show that the asymptotic behavior of the Omnès factor is fixed by the scattering phase, appearing in (42), at infinity. Namely,

$$
\Omega(s) \underset{s \rightarrow \infty}{\rightarrow} s^{-\delta(\infty) / \pi} .
$$

It follows from (29) and (34) that in general the determinant (36) satisfies the one-channel equation (37) with the phase given by $\arg \left(\operatorname{det}_{i j} S_{i j}\right) / 2$. Therefore, its asymptotic behavior (see a comment in the beginning of this section) is fixed to be

$$
D(s) \underset{s \rightarrow \infty}{\rightarrow} s^{-\arg \operatorname{det}_{i j} S_{i j}(\infty) / 2 \pi} .
$$

The degree at infinity of the determinant is given by the sum of degrees of all canonical solutions. It is obvious for a set of independent one-channel equations, corresponding to the $S$ matrix without mixing between the channels. The determinant in this case is simply a product of Omnès factors.

To use the solution (43) for finding the physical form factors, one has to know the scattering phase in (42) and the polynomial $P_{\phi}(s)$. The region where one-channel approximation could make sense is $s<4 m_{K}^{2}$. Therefore, the real

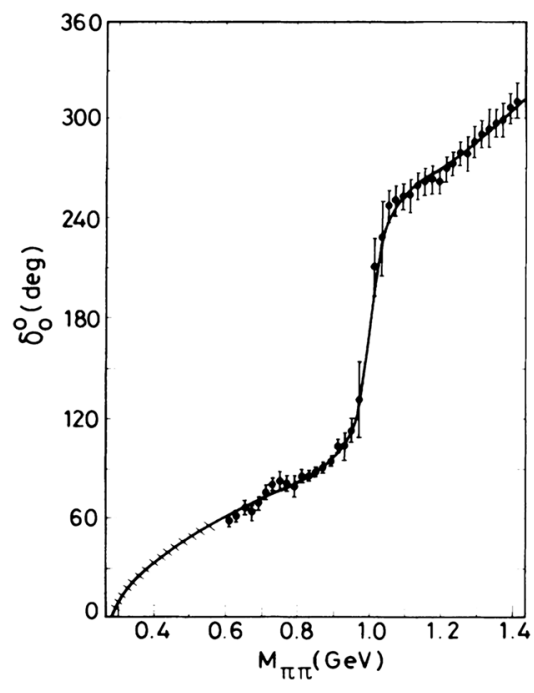

FIG. 3. Pion-pion scattering phase from Ref. [40].

pion scattering phase (Fig. 3) can be used at most up to the kaon threshold. Having no a priori favorable way to extrapolate the phase beyond this point, it is customary to extend it asymptotically to the nearest integral value in units of $\pi$ (see Fig. 4).

As for the polynomial $P_{\phi}(s)$, obviously it is not fixed by the described procedure. There is no way to even to know the degree of the polynomial without additional information. We use the minimal construction (polynomials of the lowest degree possible), satisfying constraints coming from lattice and ChPT computations, namely, the quadratic scalar radius (24) and form factors behavior at small energy (18), (19). As a result, we get for the form factor involving $u$ and $d$ quarks

$$
\Gamma_{\pi}^{1 \mathrm{ch}}(s)=m_{\pi}^{2} \Omega_{1 \mathrm{ch}}(s),
$$

which corresponds to the quadratic scalar radius $\left\langle r^{2}\right\rangle_{S, \pi}^{1 \mathrm{ch}}=$ $0.62 \mathrm{fm}^{2}$. There is an ambiguity in implementing a

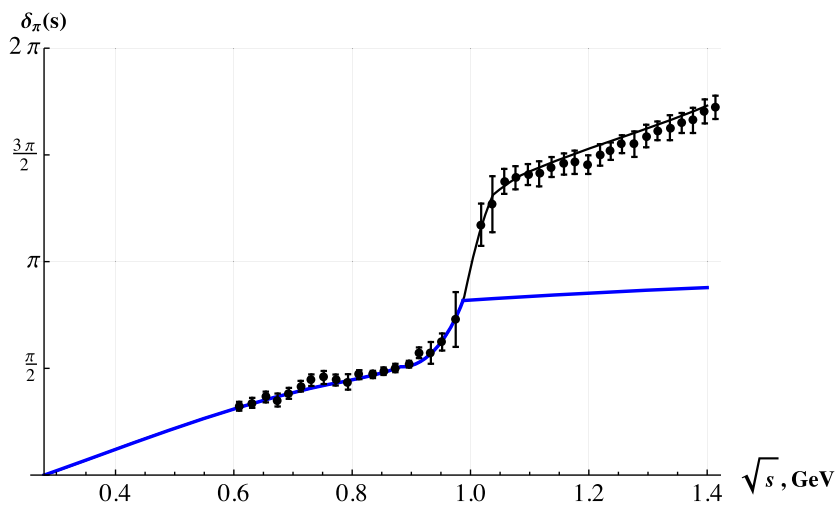

FIG. 4. Scattering phase shift: black dots correspond to the data from Ref. [40], and black curve is the interpolation. The blue line corresponds to one-channel approximation with the cutoff at kaon threshold with asymptotic value $\pi$ at infinity. 
constraint on $\theta_{\pi}(s)$. Indeed, from the expression (18), it is clear that the following two conditions are possible:

$$
\theta_{\pi}\left(-2 m_{\pi}^{2}\right)=0
$$

and

$$
\theta_{\pi}^{\prime}(0)=1
$$

These two differ by $m_{\pi}^{2} / \Lambda_{\mathrm{QCD}}^{2}$ corrections, and we adopt

$$
\theta_{\pi}^{1 \mathrm{ch}}(s)=\left(s+2 m_{\pi}^{2}\right) \Omega_{1 \mathrm{ch}}(s) .
$$

Lastly, the form factor $\Delta_{\pi}(s)$ is given by

$$
\Delta_{\pi}^{1 \mathrm{ch}}(s)=d_{F} s \Omega_{1 \mathrm{ch}}(s),
$$

with $d_{F}$ a constant (for a way to estimate its value, see below). It is clear from (44) that only one of the form factors above has the proper asymptotic $\Gamma_{1 \mathrm{ch}}(s) \underset{s \rightarrow \infty}{\rightarrow} s^{-1}$. This fact testifies that one-channel approximation does not properly describe the underlying dynamics and cannot be complete. We compare different methods in Sec. VI.

\section{B. Two channels: Numerical analysis}

For a general two-channel problem, the form factors (12a)-(12c) could be obtained by considering proper linear combinations of the canonical solutions. For normalization

$$
\Omega_{i}^{(j)}(0)=\delta_{i}^{j},
$$

where the lower index specifies the component of the corresponding solution, one could obtain the form factors reproducing ChPT results for low energy in the form

$$
\begin{aligned}
& \Gamma_{\pi}^{2 \mathrm{ch}}(s)=m_{\pi}^{2} \Omega_{1}^{(1)}+\frac{2}{\sqrt{3}} \Gamma_{K}(0) \Omega_{1}^{(2)} \\
& \Delta_{\pi}^{2 \mathrm{ch}}(s)=\frac{2}{\sqrt{3}} \Delta_{K}(0) \Omega_{1}^{(2)}
\end{aligned}
$$

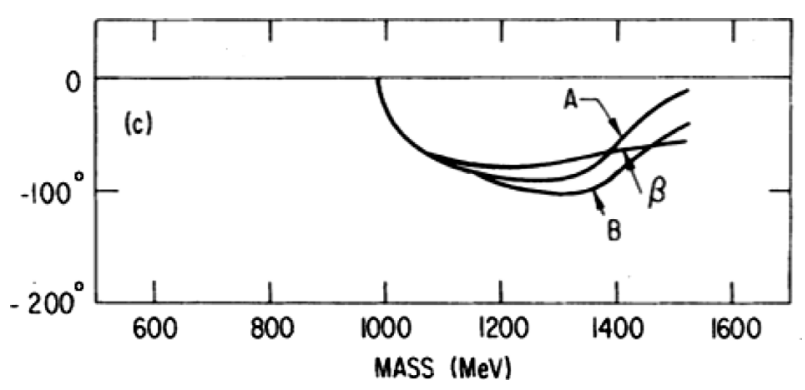

(a) Pion-kaon scattering phase from [49]. $\theta_{\pi}^{2 \mathrm{ch}}(s)=\left(2 m_{\pi}^{2}+p s\right) \Omega_{1}^{(1)}+\frac{2}{\sqrt{3}}\left(\theta_{K}(0)+q s\right) \Omega_{1}^{(2)}$,

with coefficients $p$ and $q$ related to slopes of $\theta_{\pi, K}^{\prime}(0)$.

However, as was already mentioned, an explicit analytic solution $\Omega_{i}^{(j)}(s)$ to the system (32) is generally not available. Therefore, one resorts to numerics. For the two channels, the $S$ matrix is a $2 \times 2$ unitary matrix,

$$
S=\left(\begin{array}{cc}
\eta e^{2 i \delta_{\pi}} & i \sqrt{1-\eta^{2}} e^{i\left(\delta_{\pi}+\delta_{K}\right)} \\
i \sqrt{1-\eta^{2}} e^{i\left(\delta_{\pi}+\delta_{K}\right)} & \eta e^{2 i \delta_{K}}
\end{array}\right),
$$

where $\delta_{\pi, K}$ are the scattering phases of $\pi \pi \rightarrow \pi \pi$ and $\bar{K} K \rightarrow$ $\bar{K} K$ and $\eta$ is the elasticity parameter, characterizing the mixing between the two channels. All these parameters are extracted from experimental data (see, e.g., Refs. [39,40,49] and Fig. 5).

It is precisely the mixing due to the elasticity parameter that precludes one from finding a solution analytically. Indeed, were it not for $\eta(s) \neq 1$, the system of equations (32) would factorize into two independent onechannel equations (37) that could be solved explicitly. However, one can still derive the analytic form of the determinant (36) even for a nontrivial elasticity parameter. Indeed, assuming asymptotic values for the scattering phases $\delta_{\pi}(\infty)=2 \pi, \delta_{K}(\infty)=0$, it follows immediately from (45) that the determinant of the canonical solutions decays at infinity as $s^{-2}$.

In Ref. [23], the following iterative procedure for solving the system was suggested (further developed in Refs. $[44,46])$. In the zeroth approximation, the functions $\phi_{1}^{(0)}(s), \phi_{2}^{(0)}(s)$ are initialized by constants: $\phi_{1}^{(0)}(s)=1$ and $\phi_{2}^{(0)}(s)=\lambda \in \mathbb{R}$. The real and imaginary parts of $\phi_{i}(s)$ at the step $n+1$ are computed via

$$
\begin{aligned}
\operatorname{Im} \phi_{i}^{(n+1)}(s) & =\operatorname{Re}\left[\sum_{j} T_{i j}^{*}(s) \phi_{j}^{(n)}(s) \sigma_{j}(s) \Theta\left(s-4 m_{j}^{2}\right)\right] \\
s & \in \mathbb{R}
\end{aligned}
$$

FIG. 5. Two channel $S$-matrix parameters. 
and

$$
\operatorname{Re} \phi_{i}^{(n+1)}(s)=\frac{1}{\pi} \int_{4 m_{\pi}^{2}}^{\infty} d s^{\prime} \frac{\operatorname{Im} \phi_{i}^{(n)}\left(s^{\prime}\right)}{s^{\prime}-s} .
$$

Since the two numerically obtained solutions tend to zero for $s \rightarrow \pm \infty$, it was assumed that these are the two canonical solutions, behaving as $s^{-1}$ at infinity, in agreement with $s^{-2}$ behavior of the determinant discussed above. Plugging those solutions into (51), one gets the form factors $\Gamma_{\pi}^{\mathrm{DGL}}(s), \Delta_{\pi}^{\mathrm{DGL}}(s), \theta_{\pi}^{\mathrm{DGL}}(s)$. The slope of pion form factor was taken to be $\theta_{\pi}^{\prime}(0)=1$, and other unknown parameters in (51) are obtained using $S U(3) \times S U(3)$ ChPT; in particular, the following values were chosen:

$$
\begin{aligned}
\Gamma_{K}(0) & =\frac{m_{\pi}^{2}}{2}, \quad \Delta_{K}(0)=m_{K}^{2}-\frac{m_{\pi}^{2}}{2}, \quad \theta_{K}(0)=2 m_{K}^{2}, \\
\theta_{K}^{\prime}(0) & =0.9-1.1 .
\end{aligned}
$$

As we can see, the polynomial needed to fix the lowenergy behavior of the form factor (51c) has degree 1, so the form factor $\theta_{\pi}^{\mathrm{DGL}}(s)$ does not have the proper asymptotic at infinity, which is again the signal of incompleteness of the approach (see the discussion in Ref. [23]).

We would like to bring to the readers' attention that, while the numerical procedure produces two decaying at infinity solutions, it has not been proven that so-obtained solutions are the canonical ones. There may not even exist canonical solutions all decaying at infinity; it is only the determinant (morally speaking, the product) that has a specific asymptotic behavior. It is obvious if the limit $\eta(s)=1$ is considered. In that case, the two canonical solutions, according to asymptotic of pion and kaon scattering phases, behave as $s^{-2}$ and $s^{0}$ correspondingly. By construction, the method gives access to form factors only for $s \in \mathbb{R}$; hence, it is not obvious how to check that no additional singularities (and zeros) have been generated and the two solutions are analytic in the whole complex plane with the cut.

\section{Resonance approximation}

There is a class of $T$ matrices for which explicit solutions of (32) can be constructed. Taking into account Eq. (29), we observe immediately that

$$
\phi_{i}(s)=\sum_{j} c_{j}(s) T_{j i}(s),
$$

with $c_{k}(s)$ being real functions on $s \in \mathbb{R}$, formally solves the system (31). Generally, the $T$ matrix has a left-hand cut, $^{5}$ and the expression (56) cannot represent any of the form factors. However, if there exists an analytic function

\footnotetext{
${ }^{5}$ The right-hand cut $s \geq 4 m^{2}$ corresponds to $u \leq 0$ for $t=0$ (s channel), which, due to the crossing, implies the existence of the left-hand cut $s \leq 0$.
}

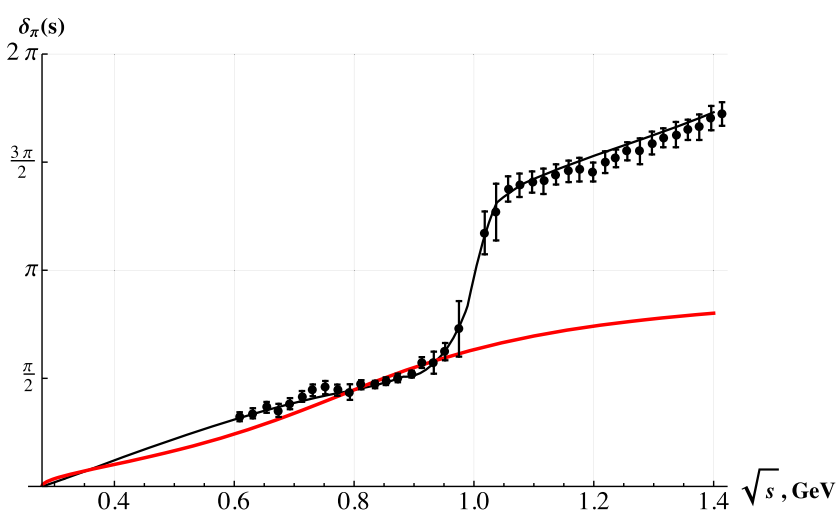

FIG. 6. One-channel resonance approximation for the scattering phase (red) with parameters from Ref. [19].

$T(s)$ with only the right-hand cut and approximating properly the scattering data (see Figs. 6 and 7), then the formula (56) provides us with a proper solution to (32).

The function considered in Refs. [19,22] has the resonance form; e.g., there are poles. However, these are, as usual, restricted to the second (unphysical) sheet. The function is built in the following way. Assuming that $T$ is symmetric, one can show using (31) that in matrix notations the relation

$$
\operatorname{Im} \boldsymbol{T}^{-1}=-\boldsymbol{\sigma}, \quad s \in \mathbb{R},
$$

with $\boldsymbol{\sigma}=\operatorname{diag}\left(\sigma_{1}(s), \sigma_{2}(s) \ldots\right)$, is satisfied. It follows from the above equation that the $T$ matrix can be formally written as

$$
\boldsymbol{T}=(\boldsymbol{A}-i \boldsymbol{\sigma})^{-1}, \quad s \in \mathbb{R},
$$

with $\boldsymbol{A}$ an arbitrary real-valued (on $\mathbb{R}$ ) matrix.

In both Refs. [19,22], the simplest choice was made,

$$
A_{i j}(s)=\frac{P_{i j}^{(1)}(s)}{\sqrt{s}},
$$

with $P_{i j}^{(1)}(s)$ being first-order polynomials. The functions $c_{k}(s)$ were also chosen to be linear, with coefficients fixed by the low-energy behavior of form factors (18) and (19).

In particular, for the one-channel $T$-matrix resonance representation

$$
T^{-1}(s)=\frac{M^{2}-s+\Gamma \sqrt{4 m_{\pi}^{2}-s}}{\sqrt{s} \Gamma},
$$

the normalized $\Omega_{\text {res1 }}(0)=1$ solution was found in Ref. [19], ${ }^{6}$

\footnotetext{
${ }^{6}$ There is a factor 2 difference in the notation for the width between our notation and the one used in the paper cited.
} 


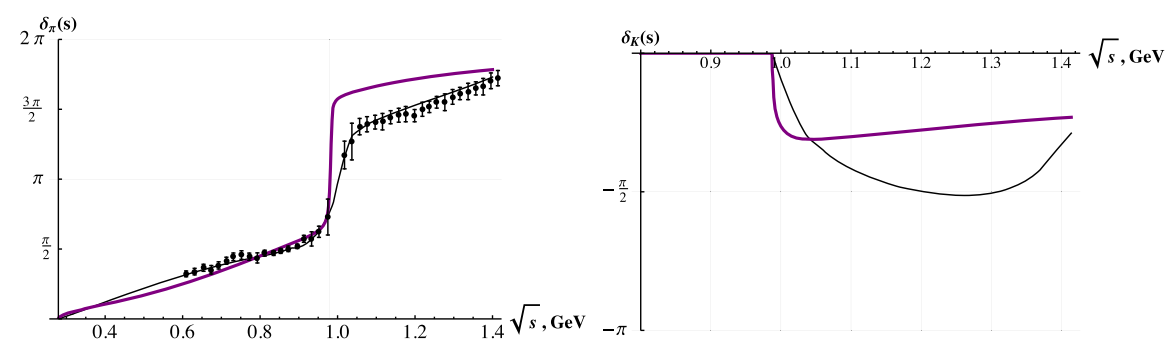

(a) $\pi \pi$ scattering phase

(b) $\bar{K} K$ scattering phase

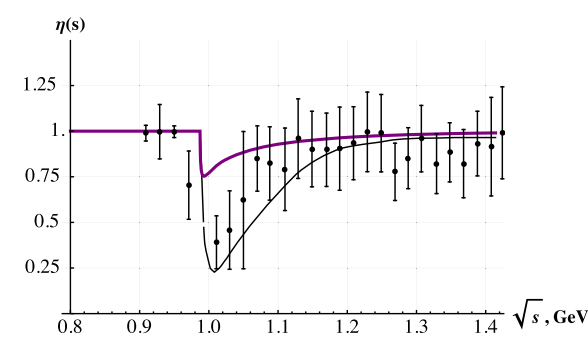

(c) Elasticity

FIG. 7. Two-channel resonance approximation (purple) for the $S$ matrix with parameters from Ref. [22].

$$
\Omega_{\mathrm{res} 1}(s)=\frac{M^{2}+2 m_{\pi} \Gamma}{M^{2}-s+\Gamma \sqrt{4 m_{\pi}^{2}-s}},
$$

with the parameters for mass and width of the resonance $M=0.8 \mathrm{GeV}$ and $\Gamma=0.65 \mathrm{GeV}$. Using that

$$
\Gamma_{\pi}^{\mathrm{res} 1}(s)=m_{\pi}^{2} \Omega_{\pi}^{\mathrm{res} 1}(s),
$$

it is straightforward to show that these parameters $\left\langle r^{2}\right\rangle_{S, \pi}=$ $0.56 \mathrm{fm}^{2}$ for the quadratic scalar radius of the pion. Meanwhile, to reproduce the value (24), one takes $M=0.85 \mathrm{GeV}$ and $\Gamma=0.8 \mathrm{GeV}$. The other two form factors are

$$
\Delta_{\pi}^{\mathrm{res} 1}(s)=0, \quad \theta_{\pi}^{\mathrm{res} 1}(s)=\left(s+2 m_{\pi}^{2}\right) \Omega_{\pi}^{\mathrm{res} 1}(s) .
$$

For two-channel resonance approximation, the $T$ matrix was chosen to have the form [22]

$$
\boldsymbol{T}^{-1}=\left(\begin{array}{cc}
\frac{M_{1}^{2}-s+\Gamma_{1} \sqrt{4 m_{\pi}^{2}-s}}{\sqrt{s} \Gamma_{1}} & -\lambda \sqrt{s} \\
-\lambda \sqrt{s} & \frac{M_{2}^{2}-s+\Gamma_{2} \sqrt{4 m_{K}^{2}-s}}{\sqrt{s} \Gamma_{2}}
\end{array}\right),
$$

with constants $M_{1}=0.87 \mathrm{GeV}, \quad \Gamma_{1}=0.7 \mathrm{GeV}, M_{2}=$ $0.92 \mathrm{GeV}$, and $\Gamma_{2}=1 \mathrm{GeV}$ now describing positions and widths of two resonances and $\lambda=-0.2$ being responsible for the coupling between the two channels. ${ }^{7}$ Knowing the form of the $T$ matrix, one builds canonical solutions normalized as in (50), and then the form factors similar to $(51){ }^{8}$

The form (59) of the matrix $\boldsymbol{A}$ is not unique, and the corresponding solution (56) can be used to approximate real form factors works inasmuch as ansatz (60) and (64) fit the experimental data. It is evident from Fig. 6 that the one-channel resonance approximation does not account for data points above the kaon threshold.

\footnotetext{
${ }^{7}$ Our notations are slightly different from the ones used in the paper cited.

${ }^{8}$ The authors of Ref. [22] follow a different procedure. They use the ChPT result directly for the amplitude (20) to impose the corresponding boundary conditions. This effectively leads to $\Delta_{\pi}(s)=0$.
}

For the two-channel resonance approximation of Ref. [22], depicted in Fig. 7, the situation is only somewhat better.

Therefore, there is no good reason to expect that the resonance approximation gives quantitatively reliable approximations for the form factors. However, since the scattering data are qualitatively reproduced, it is a good model to test other techniques and assumptions. In particular, it can be deduced that form factor $\theta_{\pi}(s)$ is almost insensitive to variations of the parameters in (51), while form factors $\Gamma_{\pi}(s)$ and $\Delta_{\pi}(s)$ depend substantially on the values of $\Gamma_{K}(0)$ and $\Delta_{K}(0)$.

\section{Modified one-channel approximation}

In this section, we present yet another approximate solution to the Muskhelishvili-Omnès problem. It is based on a specific feature of the data, namely, the fact that the elasticity parameter $\eta(s)$ appearing in the definition of the $S$ matrix (52) is consistent with $\eta=1$ almost everywhere apart from $2 m_{K} \lesssim \sqrt{s} \lesssim 1.1 \mathrm{GeV}$ [49-51] [see Fig. 5(b)]. This in turn implies that Eq. (37), i.e.,

$$
\phi_{\pi}(s)=e^{2 i \delta_{\pi}(s)} \phi_{\pi}^{*}(s)
$$

is satisfied almost everywhere except for a narrow window just above the kaon threshold. We therefore take the scalar form factor $\phi_{\pi}(s)$ in the form (42) with all the experimental data for the pion scattering phase and not only up to the kaon threshold as for the one-channel approximation discussed in Sec. VA. Above $\sqrt{s}=\Lambda_{\text {dat }}$, we extrapolate the phase in a smooth manner to $2 \pi$, which is now the nearest integral value in units of $\pi$. We call this a modified one-channel approximation. It has been previously discussed in Refs. [45-48,52,53].

As before, we use ChPT and (24) to find minimal polynomials defining form factors

$$
\begin{aligned}
& \Gamma_{\pi}^{\bmod }(s)=m_{\pi}^{2} \frac{s_{\Gamma}-s}{s_{\Gamma}} \Omega_{\text {mod }}(s), \\
& \Delta_{\pi}^{\bmod }(s)=d_{F} s \Omega_{\bmod }(s), \\
& \theta_{\pi}^{\bmod }(s)=\left(s+2 m_{\pi}^{2}\right) \Omega_{\text {mod }}(s),
\end{aligned}
$$

where $d_{F}$ is a yet unfixed constant and $\sqrt{s_{\Gamma}}=1.1 \mathrm{GeV}$. 


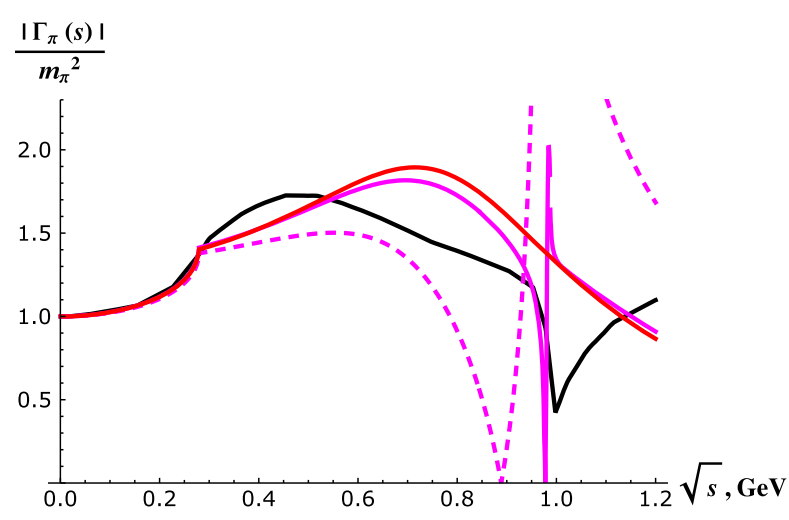

(a) One-channel resonance approximation (red), two-channel resonance approximations with parameters from [19] and [22] with $\Gamma_{K}(0)=0$ and $\Gamma_{K}(0)=4 m_{\pi}^{2}$ correspondingly (solid and dashed magenta).

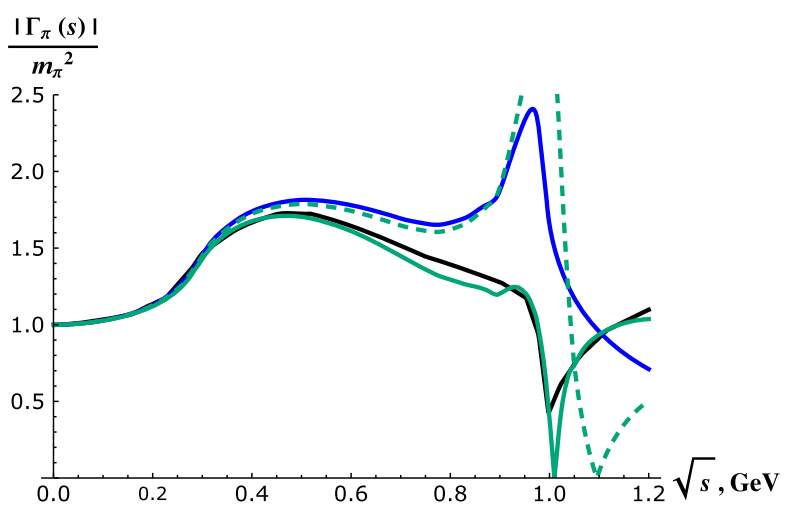

(b) One-channel approximation (blue), modified one-channel approximations with $\sqrt{s_{\Gamma}} \approx 1 \mathrm{GeV}$ and $\sqrt{s_{\Gamma}} \approx 1.1 \mathrm{GeV}$ (solid and dashed green).

FIG. 8. Absolute value of $\Gamma_{\pi}(s)$ in units of $m_{\pi}^{2}$ obtained using different methods. In both plots, the black curve is the same, taken from Ref. [46].

As we discussed in the Sec. VA, once the canonical solution is known, all other solutions are parametrized by a polynomial. Therefore, as a cross-check, the method of this section should reproduce the result of the numerical procedure from the Sec. V B; otherwise, it would not even qualify as a valid approximation. This is precisely what happens, with the only subtlety being that in order to account for the mismatch in asymptotic behaviors of $\theta_{\pi}^{\mathrm{DGL}}(s)$ and $\theta_{\pi}^{\bmod }(s)$ it is necessary to multiply the latter by a linear function,

$\theta_{\pi}^{\bmod }(s) \rightarrow \frac{s_{\theta}-s}{s_{\theta}} \theta_{\pi}^{\bmod }(s) \approx \theta_{\pi}^{\mathrm{DGL}}(s)$, with $s_{\theta}=1.3 \mathrm{GeV}^{2}$.

\section{RESULTS AND COMPARISON}

In this section, we compare results for the form factors and corresponding decay rates obtained using different methods. In Fig. 8, we plot the absolute value of the form factor $\Gamma_{\pi}(s)$ in units of $m_{\pi}^{2}$. It is evident that all methods are numerically consistent up to $s=4 m_{\pi}^{2}$. With the exception of the one-channel approximation, they all exhibit a characteristic dip in the vicinity of $\sqrt{s}=1 \mathrm{GeV}$. It could be argued that all the results are comparable up to $\sqrt{s} \approx$ $0.6-0.7 \mathrm{GeV}$ (and disagree above). Also, it is clear from Fig. 8(b) that even a slight variations of the parameter $s_{\Gamma}$ of the modified one-channel approximation, causing insignificant changes in the slope at $s=0$, or equivalently in the quadratic scalar radius of the pion, ${ }^{9}$ lead to the dramatic

\footnotetext{
${ }^{9}$ The parameter $\sqrt{s_{\Gamma}}=1.1 \mathrm{GeV}$ corresponds to the central value of (24), while $\sqrt{s_{\Gamma}}=1 \mathrm{GeV}$, effectively reproducing the result of Ref. [46], leads to $\left\langle r^{2}\right\rangle_{S, \pi}=0.57 \mathrm{fm}^{2}$, which is at the edge of the error interval.
}

difference around $\sqrt{s}=1 \mathrm{GeV}$. Similarly, we observe from Fig. 8(a) that seemingly slight variations of parameters in (51) lead to appreciable changes of the form factor.

In Fig. 9, we plot both the real and imaginary parts of the form factor $\theta_{\pi}(s)$. We see that the one-channel approximation produces the result close to that of the two-channel numerical treatment, while the deviation of the modified one-channel approximation becomes clear rather early. The reason is that the combination that reproduces the numerical analysis of Ref. [23] is not the form factor $\theta_{\pi}^{\bmod }(s)$ itself but is rather given by (67), having an additional zero. In Fig. 10, we plot the combination (67) with $\sqrt{s_{\theta}}=1.1 \mathrm{GeV}$.

In Fig. 11, we plot the form factor $\Delta_{\pi}(s)$. The modified one-channel approximation again passes the crosschecking, for it reproduces the numerical two-channel result of Refs. $[23,46]$. On the other hand, the one-channel approximation is clearly inadequate when applied to that specific form factor. Contrary to $\Gamma_{\pi}(s)$ and $\theta_{\pi}(s)$, where the one-channel approximation could be argued to-at least qualitatively - coincide with the two-channel analysis up to $\sqrt{s}=0.6 \mathrm{GeV}$, in the case of $\Delta_{\pi}(s)$, it fails already at rather small values of the center-of-mass energy. A possible reason for that can be the presence of a relatively broad $f_{0}(980)$ resonance very close to the kaon threshold, which is not captured at all by the one-channel approximation, for it does not take into account the real data above $4 m_{K}^{2}$. The disproportionate change of the form factor $\Delta_{\pi}(s)$ when compared to that of $\Gamma_{\pi}(s)$ and $\theta_{\pi}(s)$ indicates an enhanced relative significance of the $s$-quark effects for the process at hand.

Lastly, we present the results for the decay rate in Fig. 12. For both, one- and modified one-channel approximations (49) and (66b), we use $d_{F}=0.09$ from Ref. [23]. 


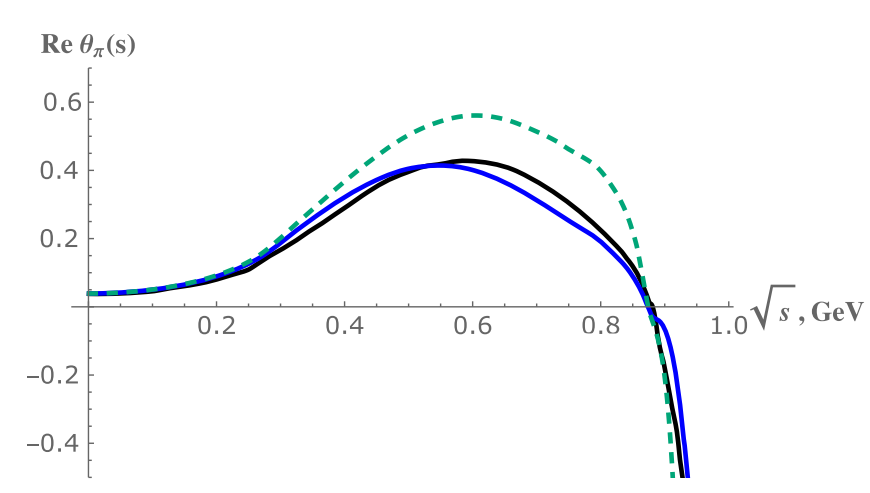

(a) Real part

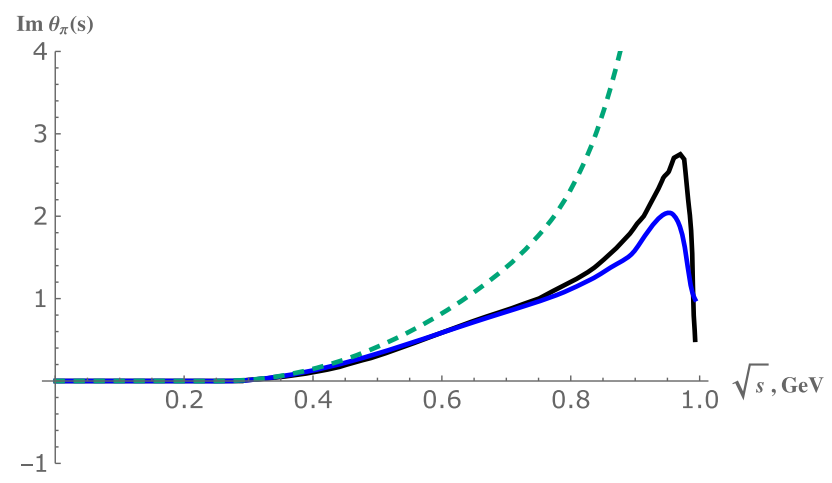

(b) Imaginary part.

FIG. 9. Real and imaginary parts of the form factor $\theta_{\pi}(s)$ for different methods: one-channel approximation (blue), modified onechannel approximation (green), and the black curve taken from Ref. [23].

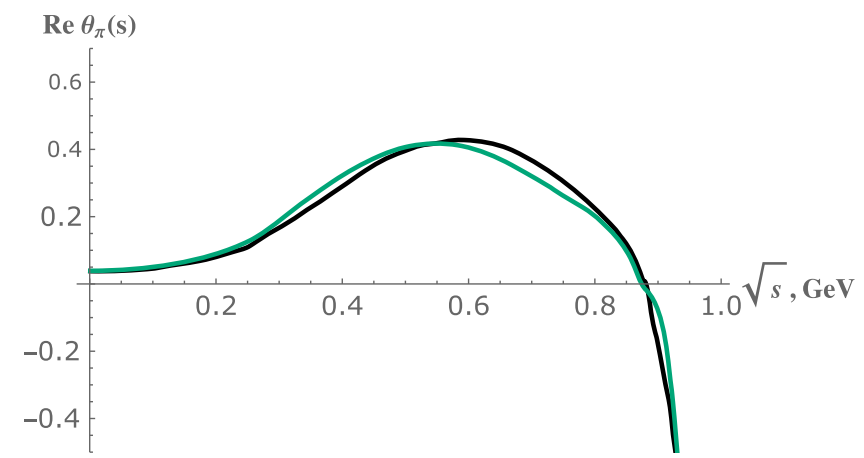

(a) Real part.

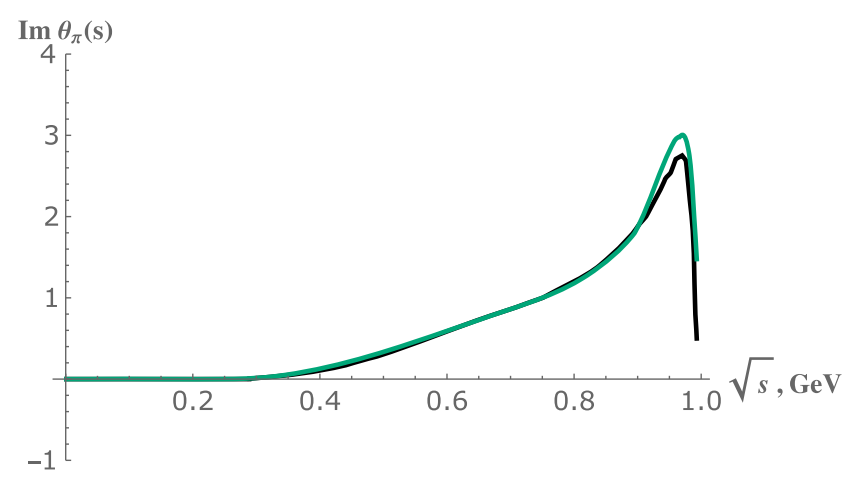

(b) Imaginary part.

FIG. 10. Reproducing the numerical result for the form factor $\theta_{\pi}(s)$ from Ref. [23] with the modified one-channel approximation by using an additional zero (67) with $s_{\theta}=1.3 \mathrm{GeV}^{2}$.

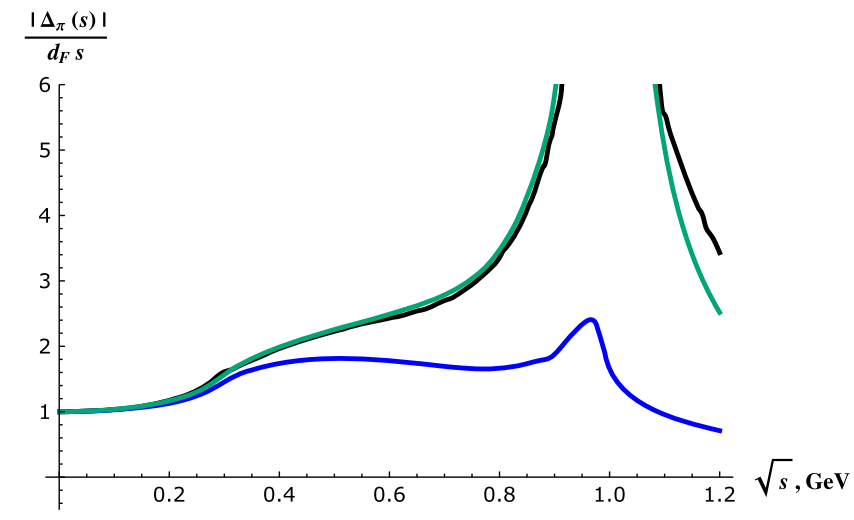

FIG. 11. Absolute value of the form factor $\Delta_{\pi}(s)$ in units of $d_{F} s$ : one-channel approximation (blue), the result for the twochannel numerical procedure from Ref. [46] (black), and the modified one-channel approximation (66) (green) coinciding with the numerical procedure up to $\sqrt{s} \approx 1 \mathrm{GeV}$.
A somewhat different value (also discussed in Ref. [23]), obtained in the limit of the heavy $s$ quark, $d_{F}=$ $2 / 29 \approx 0.068$, changes the result only slightly. As is also evidenced from plots for the form factors [Figs. 8(b), 10, and 11], the modified one-channel approximation with an additional zero (67) characterized by $\sqrt{s_{\theta}}=1.3 \mathrm{GeV}$ and $\sqrt{s}_{\Gamma}=1 \mathrm{GeV}$ reproduces numerical results of Ref. [23] [Fig. 12(a)]. It is clear that all methods (except the twochannel resonance approximation) produce results significantly different from the ChPT just above $4 m_{\pi}^{2}$. We take that as an indication that ChPT results should likely be modified at rather low energies. A similar situation occurs in the case of $\eta \rightarrow \pi^{0} \pi^{+} \pi^{-}$decay, in which the final-state interaction effects are proven to be large [31].

\section{DISCUSSION}

\section{A. Rough error estimate}

Unarguably, the two-channel numerical solution makes use of the experimental data the most; however, it does not 


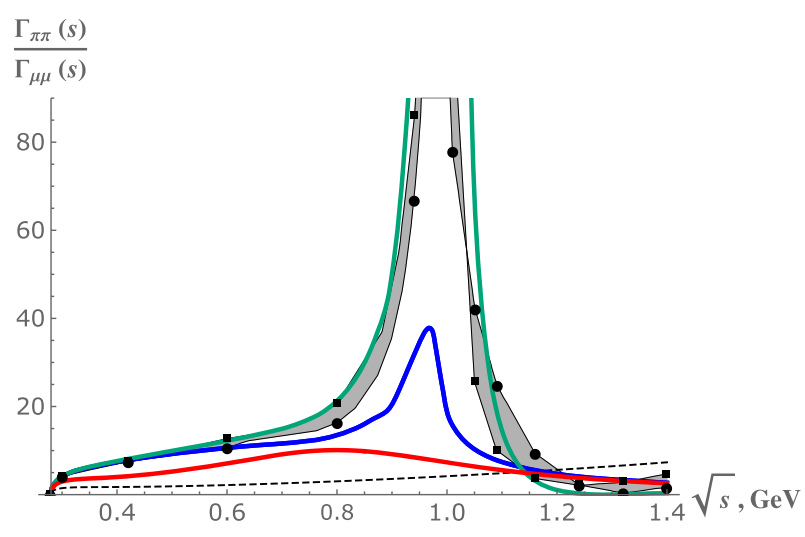

(a) The one-channel (blue), the modified onechannel approximation with $s_{\theta}=1.3 \mathrm{GeV}^{2}$ and $s_{\Gamma}=1 \mathrm{GeV}^{2}$ (green).

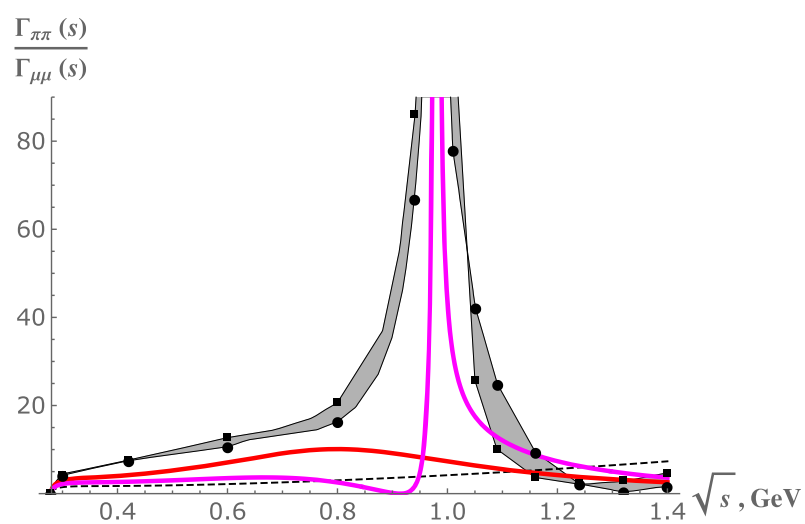

(b) Two-channel resonance approximation with parameters from [22] (purple).

FIG. 12. Branching ratio as a function of mass $m_{S}=\sqrt{s}$ : ChPT (dashed black) and one-channel resonance approximation with parameters from Ref. [19] (red). Two sets of data points taken from Ref. [23] correspond to different paramtetrizations of scattering data. The shaded grey region between the two data sets indicates uncertainty of the numerical procedure.

mean it produces reliable results. It is important to quantify the uncertainties and to figure out the domain of validity of the result. Even though examples of Sec. VI (see Fig. 8) show that form factors may appreciably depend on the parameters in (51), we will not discuss these corrections, referring the reader to Ref. [23], claiming that variations of the parameters (51) do not influence the decay rate significantly. Similarly, the dependence of the result on different inputs for the phase shifts and different interpolations of the data is not discussed, since it was addressed in Ref. [23] (see Fig. 12).

Instead, we discuss another source-the primary one from our perspective - of uncertainty, which plagues all the discussed methods. It is our ignorance about the UV dynamics, for we do not know the $S$ matrix above $\sqrt{s}=\Lambda_{\mathrm{dat}}$. The importance of scattering data above that scale is clear, for none of the methods (except the modified one-channel approximation taken at face value) produces the correct asymptotic of the form factors. This is an indication that these methods cannot present the complete picture. The role of high-energy dynamics is clearly important.

From a more technical perspective, the knowledge of the high-energy behavior of the scattering amplitudes is crucial in all the dispersion methods, involving integrals to infinity. Usually, though (say, for scattering amplitudes or cross sections), it is expected that if the UV dynamics kicks in at a relatively high-energy scale it should not affect the IR. However, for the case of form factors, not only do we not know where those effects appear, but also the information about the UV behavior of the scattering date might not be enough. The specificity of the dispersion relation in this case is that, crudely speaking, it takes into account the phase remaining oblivious to zeros (reproduced by polynomials) of the form factors. Even if we knew scattering phases at arbitrary high energies, there is no information on where to put zeros (for more, see Appendix B).

Nevertheless, neglecting the last remark, we try estimating the error coming only from changes in scattering data at higher energies. To do that, instead of solving the system of two coupled channels with a different asymptotic for scattering phases, it is easier to consider the approximation to the solution provided by the modified one-channel approximation (66) and (67), which is amenable to analytic treatment. The scattering phase is known up to $s=\Lambda_{\text {dat }}^{2}$. We assumed that at infinity it asymptotes to $2 \pi$. The ratio between the Omnès factor obtained with the help of the so-defined phase (42) and the physical one is given by

$$
\frac{\Omega_{\pi}(s)}{\Omega_{\pi}^{\text {phys }}(s)}=\exp \left[\frac{s}{\pi} \int_{\Lambda_{\mathrm{dat}}^{2}}^{\infty} \frac{d s^{\prime}}{s^{\prime}} \frac{\delta_{\pi}\left(s^{\prime}\right)-\delta_{\pi}^{\text {phys }}\left(s^{\prime}\right)}{s^{\prime}-s-i \varepsilon}\right] .
$$

Focusing only on situations in which the asymptotic behavior is different, it is easy to estimate the correction at small $s$,

$$
\Omega_{\pi}^{\text {phys }}(s) \approx \Omega_{\pi}(s)\left(1+c \frac{s}{\Lambda_{\text {diff }}^{2}}\right)
$$

where $\Lambda_{\text {diff }}$ is the scale in which the values of $\delta_{\pi}(s)$ and $\delta_{\pi}^{\text {phys }}(s)$ differ by $\pi$ (e.g., where a new resonance comes into play) and $c$ is an order- 1 constant.

\section{B. Domain of validity}

Armed with (69), we can estimate how reliable the results are at a specific scale. The benchmark for errors is set by other corrections: the derivation of Sec. II is performed at the leading order in the heavy quark mass 


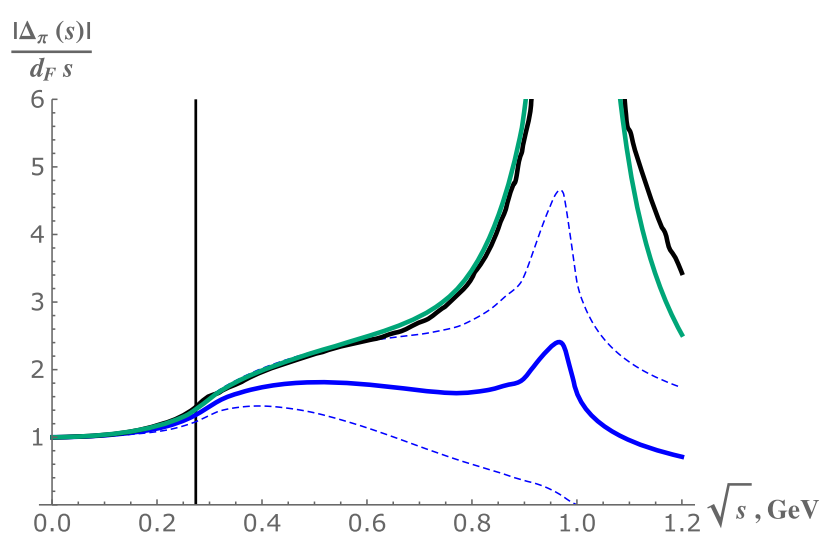

(a) $\Delta_{\pi}(s)$ form factor.

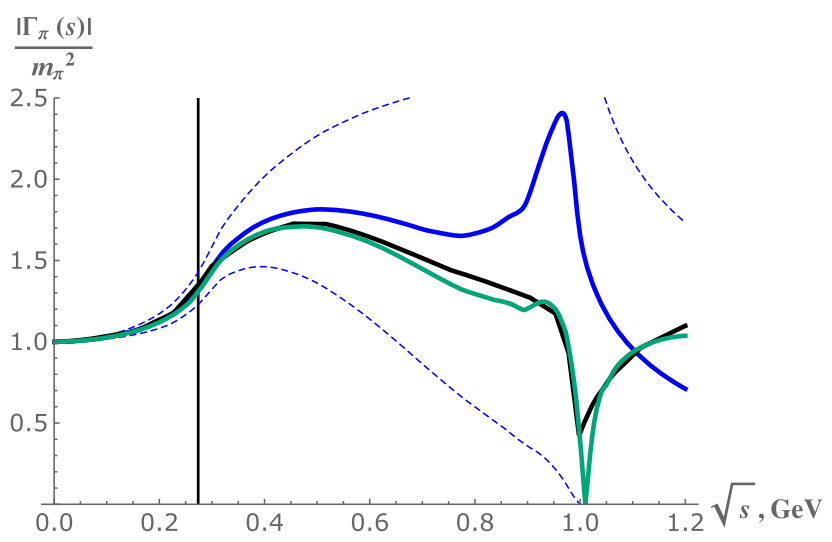

(b) $\Gamma_{\pi}(s)$ form factor.

FIG. 13. Error estimates: results for $\Gamma_{\pi}$ (a) and $\Delta_{\pi}$ (b) in the one-channel approximation (blue) with a relative error (69) shown in dashed blue. Two-channel numerical results are shown in black from Ref. [46]. The modified one-channel approximation with an additional zero is shown in green. The black vertical line corresponds to $\sqrt{s}=2 m_{\pi}$.

$m_{c}^{-2}$ and the strong coupling $\alpha_{s}$. Next-to-leading corrections can be found correspondingly in Refs. [24,54]. The relative correction due to QCD loops is estimated to be $5 \%-10 \%$ [54]. At the same time, we see from Fig. 12 that the discrepancy in the decay rate between different solutions obtained in Ref. [23] does not exceed $30 \%$ up to $1 \mathrm{GeV}$. Therefore, the relative error of $10 \%-15 \%$ for form factors seems to be a good starting point, ${ }^{10}$ and the scale $\sim \Lambda_{\text {diff }} / 2$ is where the naively estimated corrections due to UV phase shifts (69) exceed this benchmark.

For an example, we see from Fig. 13(a) that the behavior of the $\Delta_{\pi}(s)$ form factor is perfectly consistent with (69) with $c=1$ and $\Lambda_{\text {diff }}^{2}=4 m_{K}^{2}$. The reason for that is the occurrence of the resonance $f_{0}(980)$, leading to a sharp increase in the scattering phase around the kaon threshold. At the same time, Fig. 13(b) shows that, even though the actual two-channel result lies within corrections dictated by (69), the latter ones are obviously an overestimate, and the scale in which one- and two-channel results deviate from each other is larger than is expected naively, which effectively means that in this case $c<1$. That in turn leads to a better agreement between the results for the decay rate.

To estimate how wide the domain of validity is for two-channel numerical analysis of Ref. [23] (equivalently, the modified one-channel approximation), it is necessarily to know whether, similar to $f_{0}(980)$, resonances above $\Lambda_{\text {dat }}$ play a significant role for scattering phases. While $f_{0}(1370)$ is rather wide and we may not see its effect, there are claims that $f_{0}(1500)$ is visible in the scattering data [55]. At the same time, it is unknown whether the

\footnotetext{
${ }^{10}$ As discussed above, in the interval $16 m_{\pi}^{2}<s<\Lambda_{\text {dat }}^{2}$, the restriction of the $S$ matrix to two channels is only approximately unitary. Mixing with multipion states leads to about $6 \%$ corrections according to Ref. [45].
}

resonances $f_{0}(1710), f_{0}(2110), f_{0}(2200)$, and $f_{0}(2330)$, listed in Ref. [56], affect the scattering phase. As a result, if indeed $f_{0}(1500)$ affects the scattering phase, the cutoff $\Lambda_{\text {diff }}$ would be given by $1.5 \mathrm{GeV}$. On the other hand, if none of those resonances changes the behavior of the scattering phase, the cutoff will be at least $2.5 \mathrm{GeV}$.

We plot the decay rate as a function of the scalar mass $m_{S}$ with the corresponding corrections in Fig. 14. We note that the error estimate (69) should be considered as an upper bound, for as in Fig. 13(b), the actual corrections, with the dynamics of all channels taken into account, can be noticeable reduced. The reason is that changing the asymptotic of the scattering phase by $\pi$ necessitates using a polynomial of one degree higher (introducing an additional zero) to preserve the correct asymptotic behavior of the form factor at infinity, unless it was incorrect in the first place. The relative correction results in the two effects (partially) canceling each other:

$$
\left(1+c \frac{s}{\Lambda_{\mathrm{diff}}^{2}}\right)\left(1-\frac{s}{s_{0}}\right)=1+c \frac{s}{\Lambda_{\mathrm{diff}}^{2}}-\frac{s}{s_{0}}
$$

We see that the error depends on where the position $s_{0} \geq$ $\Lambda_{\text {diff }}^{2}$ of the additional zero is. If it is very close to the cutoff $s_{0} \approx \Lambda_{\text {diff }}^{2}$ - what happens for $\Gamma_{\pi}^{\bmod }(s)$ — the error is significantly reduced, while if it lies far in the UV, or the behavior of the form factor was incorrect before modifying the phase-hence, there is no need to use a different polynomial with an extra zero [this is precisely the case for $\left.\Delta_{\pi}^{\text {lch }}(s)\right]$ — the error is given by (69).

\section{Experimental data for $\psi^{\prime}$ and $\Upsilon^{\prime}$ decays}

Even though all methods discussed (taken at face value) produce results substantially different from the ChPT, there are arguments in favor of extrapolating the ChPT up to 


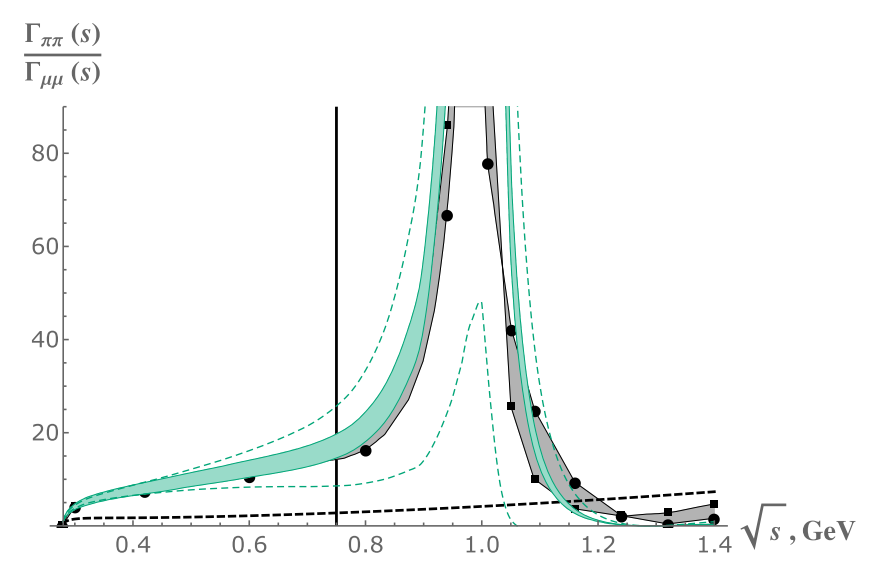

(a) $\Lambda_{\text {diff }}=1.5 \mathrm{GeV}$.

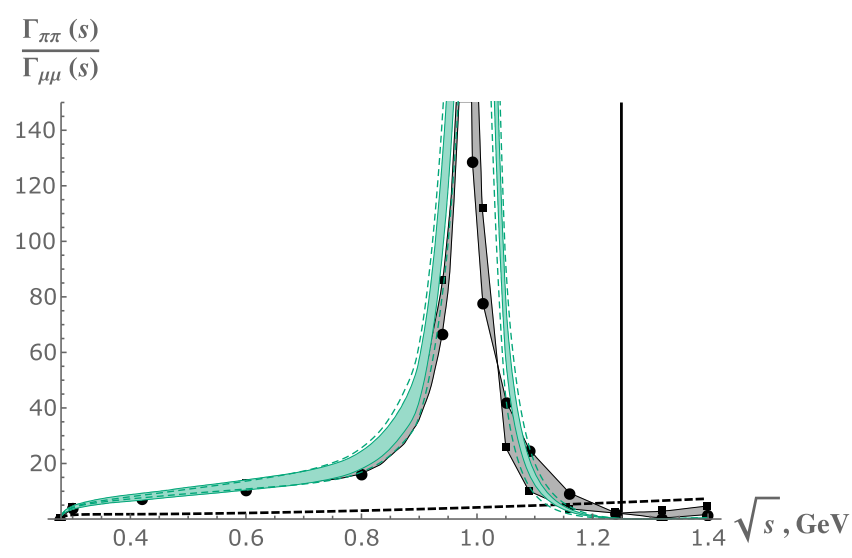

(b) $\Lambda_{\text {diff }}=2.5 \mathrm{GeV}$

FIG. 14. Branching ratio: data points and shaded grey regions are the same as in Fig. 12; ChPT result (dashed black) and the modified one-channel approximation (green) with $d_{F}=0.09, s_{\theta}=1.3 \mathrm{GeV}^{2}$, and $s_{\Gamma}=1 \mathrm{GeV}^{2}$ with shaded green regions indicating $30 \%$ error bars. Dashed lines correspond to naively corrected results with a factor $\left(1 \pm s / \Lambda_{\text {diff }}^{2}\right)$ according to (69); black vertical lines show where the leading correction becomes $100 \%$.

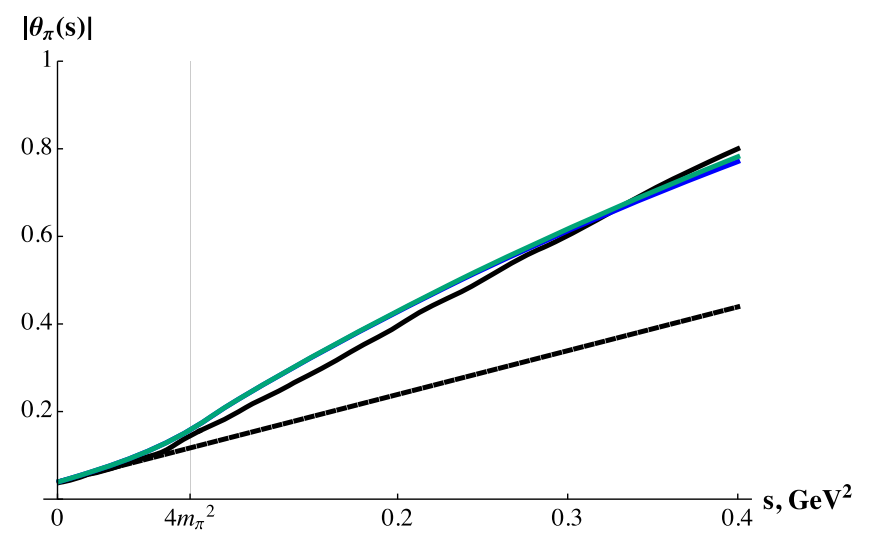

FIG. 15. Approximately linear behavior of $\theta_{\pi}(s)$ : One-channel solution (blue), modified one-channel solution (green), twochannel numeric solution (solid black), and ChPT (black dashed).

$\sqrt{s}=0.6 \mathrm{GeV}$. In Ref. [18], it is assumed that the decay rate $\Gamma_{\pi \pi}$ is dominated by the form factor $\theta_{\pi}(s)$. At the same time, according to the data from $\Upsilon^{\prime} \rightarrow \Upsilon^{\prime} \pi \pi$ [57] and $\psi^{\prime} \rightarrow$ $\psi \pi \pi[58,59]$ decays, the form factor $\theta_{\pi}(s)$ behaves linearly in the relevant interval of energies [60-62]. Thus, it is concluded that there is no deviation from ChPT prediction at these energies. However (see Fig. 15), the behavior of the form factor $\theta_{\pi}(s)$ obtained via dispersion relations is also close to linear. It would be helpful to find the slope of the form factor in the interval $2 m_{\pi} \leq \sqrt{s} \lesssim 0.6 \mathrm{GeV}$ using the data for mentioned decays. That could potentially resolve the issue and will be considered elsewhere.

\section{CONCLUSION}

In this work, we have revisited the problem of computing the hadronic decay width of a Higgs-like scalar with mass of order $m_{S} \sim 1 \mathrm{GeV}$. At these energies, decays $S \rightarrow \pi \pi$ or $S \rightarrow \bar{K} K$ cannot be adequately described by ChPT. It was previously demonstrated [23] that nonperturbative methods, reconstructing the form factors from the data on meson scattering, yield the results that are indeed different from the leading-order ChPT calculations [18], significantly enhancing the decay rate starting from several hundreds of mega-electron-volts. The tradeoff is that the data on meson scattering, used as an input, should be provided for arbitrarily high energies, which is of course not possible. In practice, having the data only up to a certain energy produces an approximation to the exact function. The assessment of the uncertainties of the estimate [23] has never been performed before.

The results of this work are summarized as follows. We reconsidered the nonperturbative evaluation of the hadronic decay width, paying special attention to the uncertainties of the method. Even though above $1 \mathrm{GeV}$ there are at least two relevant channels $(\pi \pi$ and $\bar{K} K)$, in this paper, we used the (modified) one-channel approximation to reproduce the results of the two-channel numerical analysis of Ref. [23]. This one-channel approximation works well because the elasticity parameter, controlling the coupling between the two channels, differs from 1 only in a narrow region above the kaon threshold. That effectively renders the two channels independent outside of this narrow region. The approximate one-channel solution is advantageous as it is known analytically for any scattering phase. The corresponding parameters reproducing Ref. [23] are $d_{F}=0.09$, $s_{\theta}=1.3 \mathrm{GeV}^{2}$, and $s_{\Gamma}=1 \mathrm{GeV}^{2}$. Therefore, it is straightforward to estimate corrections coming from different UV behaviors of the scattering phase. We analyzed different scenarios, and our main result is presented in Fig. 14, showing the upper bound for the error coming from the unknown UV dynamics. 
It seems unarguable that the leading ChPT result proves to be inadequate in describing the process (even in a close vicinity of the $\pi \pi$ threshold). The reason is (see also Ref. [23]) the presence of a rather broad $f_{0}(980)$ resonance. However, we should stress once again (see also comments in Sec. VII and Appendix B) that our analysis is performed neglecting the structure of zeros for all form factors. It is not inconceivable that those can lead to significant changes of form factors, and additional investigation is in order.

\section{ACKNOWLEDGMENTS}

We are extremely grateful to Professors G. Colangelo, M. Shaposhnikov, and M. Voloshin for discussions and valuable comments. However, any mistakes that may be in the present paper are solely our responsibility. The work of A.M. is supported by the ERC-AdG-2015 Grant No. 694896 and the Swiss National Science Foundation Ambizione grant.

\section{APPENDIX A: EXTRAPOLATING THE LEADING ChPT RESULT}

The estimate of the scale where the leading ChPT result breaks down is based on the paper [63] and done as follows. It is assumed that the decay rate is dominated by $\theta(s)$ and is thus related to the spectral density

$$
\rho\left(q^{2}\right)=(2 \pi)^{4} \sum_{n}\left|\left\langle 0\left|\theta_{\mu}^{\mu}(0)\right| n\right\rangle\right|^{2} \delta^{4}\left(q-q_{n}\right),
$$

evaluated at $q^{2}=m_{S}^{2}$. The spectral density is given by the imaginary part of the two-point correlation function of the energy-momentum tensor (5), which in turn in the chiral limit is expressed in terms of the gluon field strength, namely,

$$
\begin{aligned}
\rho\left(q^{2}\right) & =2 \operatorname{Im} \Pi\left(q^{2}\right) \\
& =2 i \int d^{4} x e^{i q x}\left\langle\mathrm{~T} \frac{\beta(\alpha)}{4 \alpha} G^{2}(x) \frac{\beta(\alpha)}{4 \alpha} G^{2}(0)\right\rangle .
\end{aligned}
$$

At low energies, spectral density (A1) is saturated by Goldstone bosons and can be computed taking into account (18),

$$
\rho(s)=\frac{N_{\ell}^{2}-1}{16 \pi} s^{2}+O\left(s^{3}\right),
$$

with $N_{l}$ being the number of light quarks. On the other hand, it follows (see Ref. [63]) that the following sum rule is satisfied,

$$
\int \frac{d s}{s}\left[\rho(s)-\rho_{\text {pert }}(s)\right]=b \alpha\left\langle G^{2}(0)\right\rangle,
$$

where $\rho_{\text {pert }}(s)$ is the perturbative contribution, the counterpart on the rhs of which is implicitly present in the form of the renormalization of $\left\langle G^{2}(0)\right\rangle$ needed to make it finite. It is expected that for large enough energies $\rho(s)$ is accurately represented by $\rho_{\text {pert }}(s)$. Therefore, the integral (A4) is saturated at low energies. Thus, doing the integral with the leading-order (LO) approximation (A3) and taking into account the value for $\left\langle G^{2}(0)\right\rangle$ from Refs. [64,65] allows one to estimate the cutoff where the true value of $\rho(s)$ substantially deviates from the LO (A3), which happens to be around $1.4 \mathrm{GeV}$.

The reasoning presented above is somewhat reminiscent of that for the fine-tuning problem and certainly gives an upper bound for when the LO approximation breaks down. However, it may happen earlier. Higher order in momentum corrections may kick in to bring the scale, in which the approximation cannot be trusted anymore, down.

\section{APPENDIX B: MODELING PHASE}

In this Appendix, we demonstrate how different UV behaviors of the scattering phase affect form factors. For that, we consider the following hypothetical situation. We assume that the scattering phase is known up to $1.5 \mathrm{GeV}$, and it is extrapolated to $2 \pi$ above. ${ }^{11}$ The actual behavior of the physical phase at the same time is modeled by three different scenarios depicted in Fig. 16.

The first model [Fig. 16(a)] assumes only one relatively narrow and far (occurring at $2.5 \mathrm{GeV}$ ) resonance. The corresponding Omnès factor, obtained using the formula (43), is depicted in Fig. 17. We see that the effect of the phase change is significant only in the vicinity of the resonance. Moreover, the leading-order correction (69) almost perfectly reproduces the result up to $1.6-1.7 \mathrm{GeV}$.

As the resonance moves closer $(1.7 \mathrm{GeV})$, the model in Fig. 16(b), it affects appreciably the Omnès factor [see Fig. 18(a)] already around $1 \mathrm{GeV}$, and the correction (69) accounts for the difference at most up to $1.1-1.2 \mathrm{GeV}$. Yet different behavior is captured by the model with two overlapping (at 1.7 and $1.9 \mathrm{GeV}$ ) resonances [Fig. 16(c)]. The Omnès factor, as it is seen in Fig. 18(b), is affected even more. The correction (69) should take into account both resonances now, leading to the effective $\tilde{\Lambda}_{\text {diff }}=1.2 \mathrm{GeV}$.

What these three models show is that the Omnès factor obtained with incomplete data definitely cannot be extended above the corresponding cutoff $\Lambda_{\text {diff }}$. At the same time, its low-energy behavior is not affected much by the UV behavior of the scattering phase.

Now, we turn to the corresponding expression for the form factor $\phi(s)$. Assuming that it is normalized to 1 at $s=0$ and has the asymptotic behavior at large energies $1 / s^{2}$, we see that for models with one and two additional resonances it has the following exact form correspondingly,

\footnotetext{
${ }^{11}$ The phase is chosen to qualitatively reproduce the real-world behavior.
} 


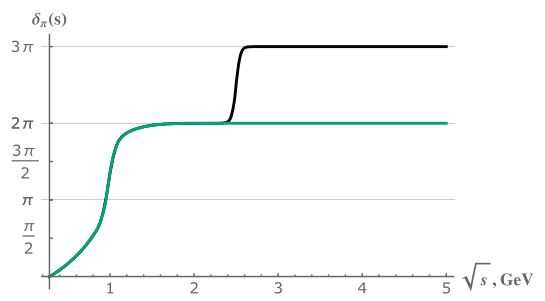

(a) Resonance at $\Lambda_{\text {diff }}=$ $2.5 \mathrm{GeV}$.

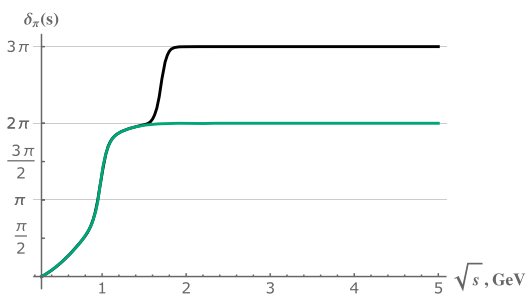

(b) Resonance at $\Lambda_{\text {diff }}=$ $1.7 \mathrm{GeV}$.

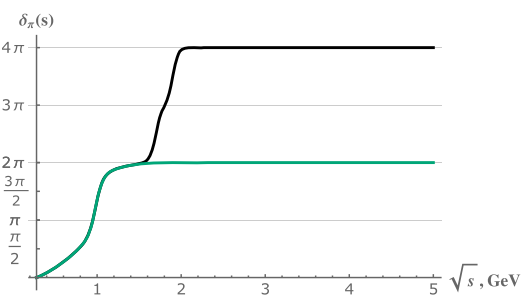

(c) Resonances at $1.7 \mathrm{GeV}$ and $1.9 \mathrm{GeV}$.

FIG. 16. Scattering phase behavior modeled by several resonances: "experimental" data (green) and physical phase (black).

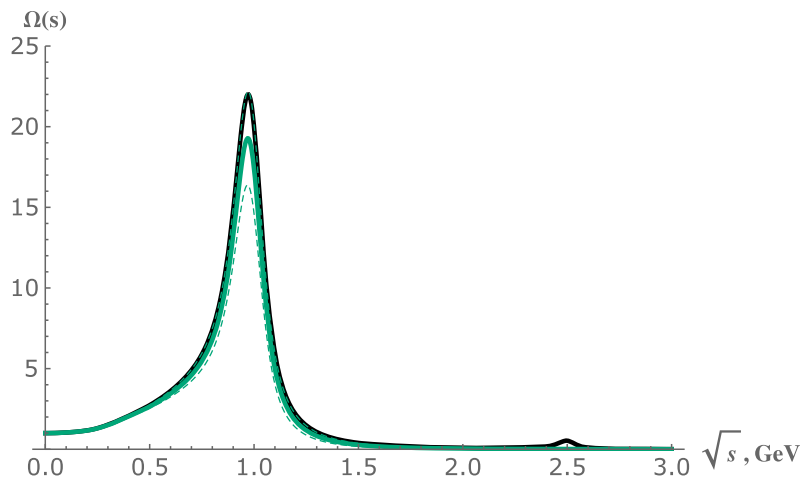

(a) $\Lambda_{\text {diff }}=2.5 \mathrm{GeV}$.

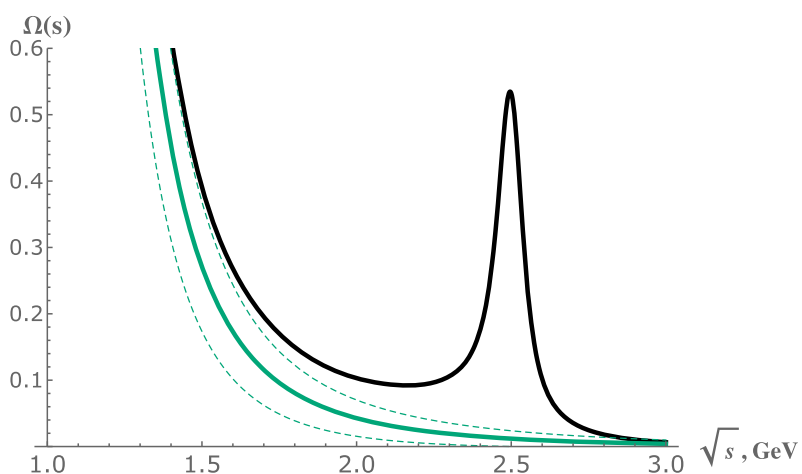

(b) $\Lambda_{\text {diff }}=2.5 \mathrm{GeV}$ : this is a zoomed in version of panel (a).

FIG. 17. Omnès factors for the model in Fig. 16(a): corresponding to experimental data (green) and physical (black). Dashed lines represent naive corrections $\left(1 \pm s / \Lambda_{\text {diff }}^{2}\right.$ ) according to (69), with $\Lambda_{\text {diff }}=2.5 \mathrm{GeV}$.

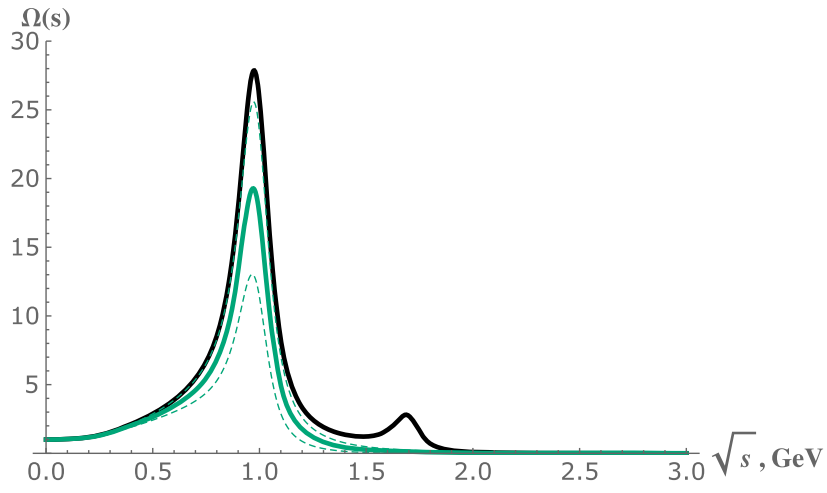

(a) $\Lambda_{\text {diff }}=1.7 \mathrm{GeV}$.

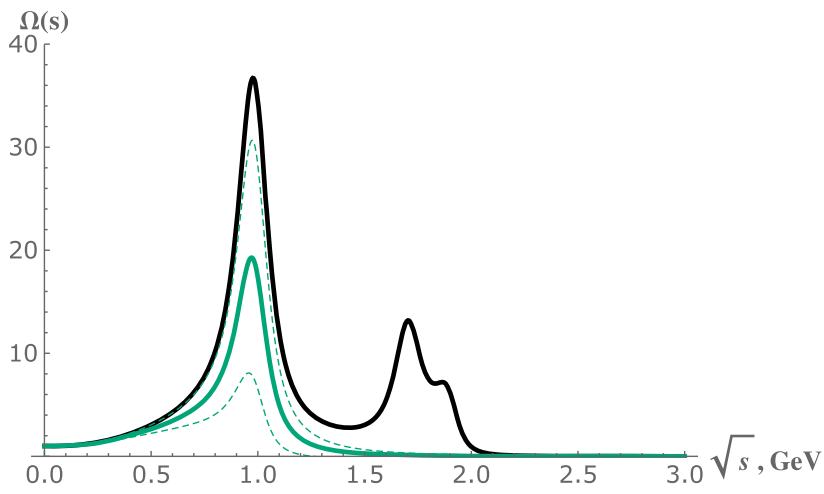

(b) Effective $\tilde{\Lambda}_{\text {diff }}=1.3 \mathrm{GeV}$.

FIG. 18. Omnès factors for model in Figs. 16(b)and 16(c): corresponding to experimental data (green) and actual (black). Dashed lines correspond to naively corrected results $\left(1 \pm s / \Lambda_{\text {diff }}^{2}\right)$ according to (69).

$$
\begin{aligned}
& \phi_{\text {phys }}(s)=\left(1-\frac{s}{s_{1}^{\text {phys }}}\right) \Omega_{\text {phys }}(s), \quad \text { and } \\
& \phi_{\text {phys }}(s)=\left(1-\frac{s}{s_{1}^{\text {phys }}}\right)\left(1-\frac{s}{s_{2}^{\text {phys }}}\right) \Omega_{\text {phys }}(s),
\end{aligned}
$$

with certain unknown parameters $s_{1,2}^{\text {phys }}$, while, due to insufficiency of the data, we approximate the form factor by

$$
\phi(s)=\Omega(s) .
$$




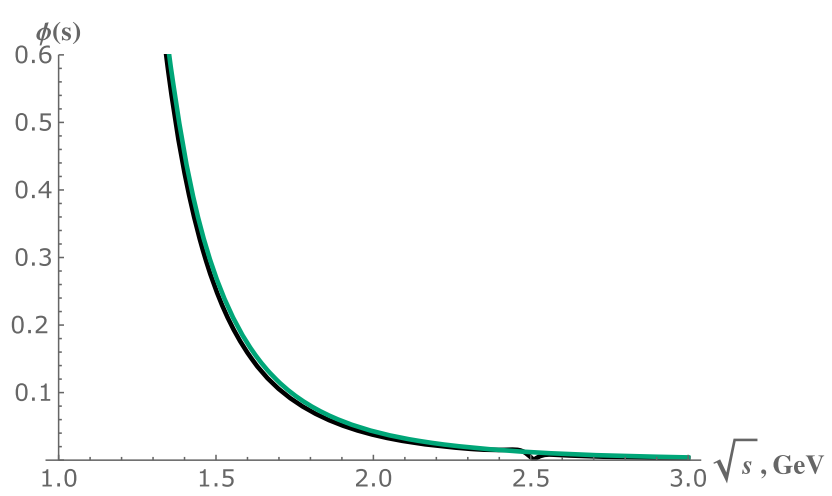

(a) $s_{1}^{\text {phys }} \approx 2.5^{2} \mathrm{GeV}^{2}$.

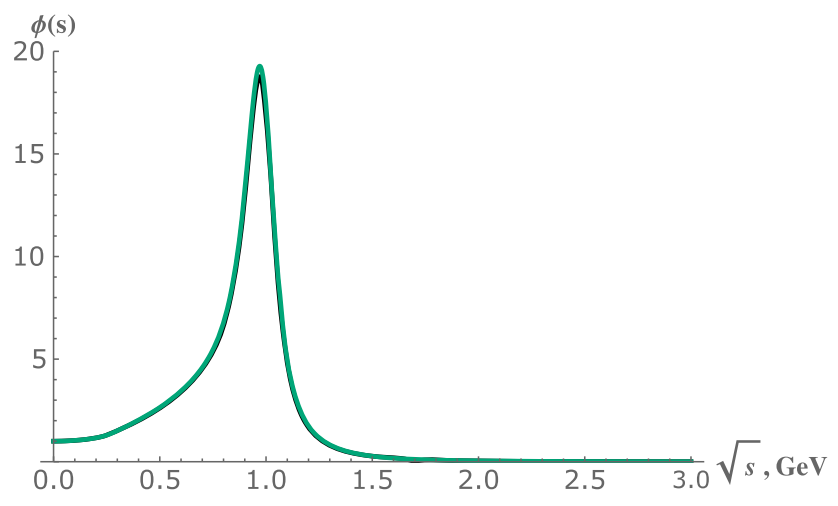

(c) $s_{1}^{\text {phys }} \approx 1.7^{2} \mathrm{GeV}^{2}$.

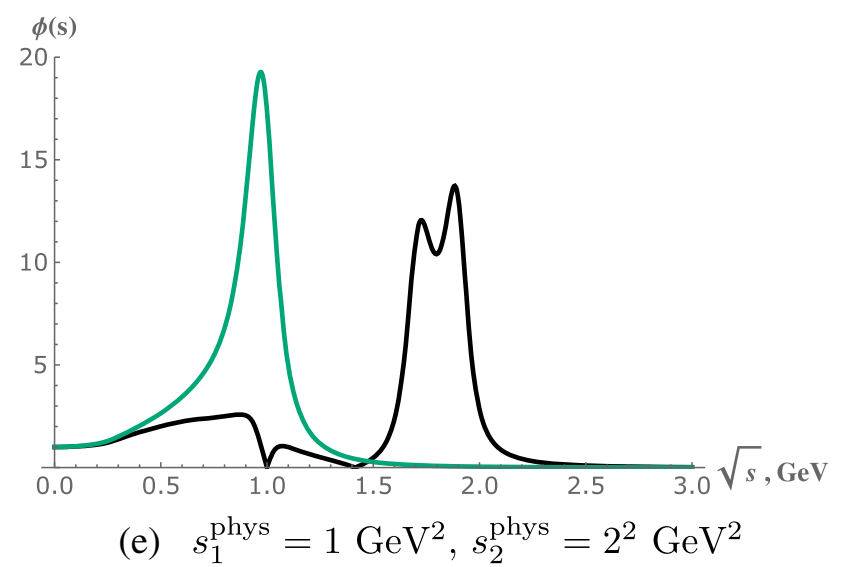

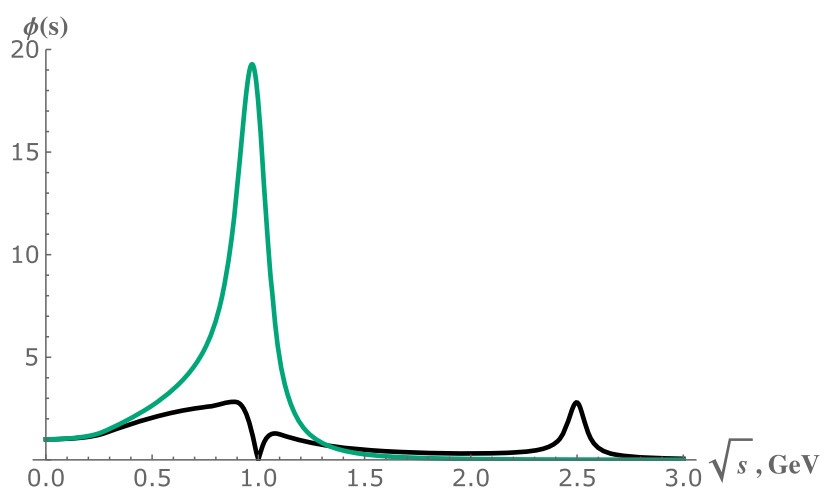

(b) $s_{1}^{\text {phys }}=1 \mathrm{GeV}^{2}$.

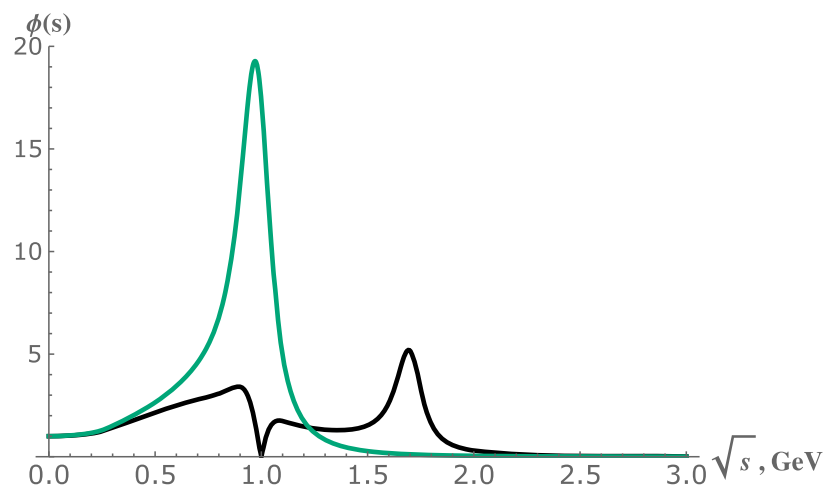

(d) $s_{1}^{\text {phys }}=1 \mathrm{GeV}^{2}$.

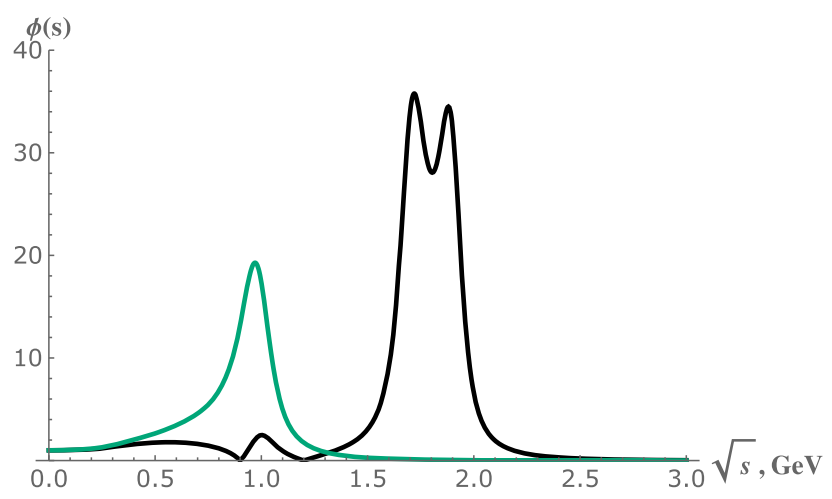

(f) $s_{1}^{\text {phys }}=0.9^{2} \mathrm{GeV}^{2}, s_{2}^{\text {phys }}=1.2^{2} \mathrm{GeV}^{2}$

FIG. 19. The form factor for different models: panels (a) and (b) correspond to Fig. 16(a), panels (c) and (d) correspond to Fig. 16(b), and panels (e) and (f) correspond to Fig. 16(c). Black curves represent the physical form factor (B1) for different values of $s_{1,2}^{\text {phys }}$, while green is the approximation from (B2).

As we see from (70) accuracy of the approximation can be enhanced if an additional zero occurs close to the resonance. From Figs. 19(a) and 19(c) illustrate such a scenario. On the other hand, for a generic situation, the form factor is not reproduced correctly. 
[1] T. Asaka, S. Blanchet, and M. Shaposhnikov, The nuMSM, dark matter and neutrino masses, Phys. Lett. B 631, 151 (2005).

[2] T. Asaka and M. Shaposhnikov, The nuMSM, dark matter and baryon asymmetry of the universe, Phys. Lett. B 620, 17 (2005).

[3] M. Shaposhnikov, Is there a new physics between electroweak and Planck scales? in Astroparticle Physics: Current Issues, 2007 (APCI07) Budapest, Hungary, 2007 (2007). http://inspirehep.net/record/759157/files/arXiv:0708.3550 .pdf.

[4] F. Bezrukov, M. Yu. Kalmykov, B. A. Kniehl, and M. Shaposhnikov, Higgs boson mass and new physics, J. High Energy Phys. 10 (2012) 140; 10 (2012) 275.

[5] F. Bezrukov and M. Shaposhnikov, Why should we care about the top quark Yukawa coupling?, Zh. Eksp. Teor. Fiz. 147, 389 (2015) [J. Exp. Theor. Phys. 120, 335 (2015)].

[6] B. Patt and F. Wilczek, Higgs-field portal into hidden sectors, arXiv:hep-ph/0605188.

[7] T. Flacke, C. Frugiuele, E. Fuchs, R. S. Gupta, and G. Perez, Phenomenology of relaxion-Higgs mixing, J. High Energy Phys. 06 (2017) 050.

[8] P. Mermod (SHiP Collaboration), Hidden sector searches with SHiP and NA62, in 2017 International Workshop on Neutrinos from Accelerators (NuFact17) Uppsala University Main Building, Uppsala, Sweden, 2017 (2017), DOI: 10.22323/1.295.0139.

[9] B. Dbrich (NA62 Collaboration), Searches for very weaklycoupled particles beyond the standard Model with NA62, in Proceedings of the 13th Patras Workshop on Axions, WIMPs and WISPs, (PATRAS 2017): Thessaloniki, Greece, 2017 (2018), pp. 145-148, DOI: 10.3204/DESY-PROC2017-02/dobrich_babette.

[10] S. Alekhin et al., A facility to search for hidden particles at the CERN SPS: The SHiP physics case, Rep. Prog. Phys. 79, 124201 (2016).

[11] M. Anelli et al. (SHiP Collaboration), A facility to search for hidden particles (SHiP) at the CERN SPS, arXiv:1504.04956.

[12] D. Curtin and M. E. Peskin, Analysis of long lived particle decays with the MATHUSLA Detector, Phys. Rev. D 97, 015006 (2018).

[13] J. A. Evans, Detecting hidden particles with MATHUSLA, Phys. Rev. D 97, 055046 (2018).

[14] J. L. Feng, I. Galon, F. Kling, and S. Trojanowski, Dark Higgs bosons at the ForwArd Search ExpeRiment, Phys. Rev. D 97, 055034 (2018).

[15] V. V. Gligorov, S. Knapen, M. Papucci, and D. J. Robinson, Searching for long-lived particles: A compact detector for exotics at LHCb, Phys. Rev. D 97, 015023 (2018).

[16] A. Berlin, S. Gori, P. Schuster, and N. Toro, Dark sectors at the Fermilab SeaQuest experiment, Phys. Rev. D 98, 035011 (2018).

[17] A. A. Anselm, N. G. Uraltsev, and V. A. Khoze, Higgs particles, Usp. Fiz. Nauk 145, 185 (1985) [Sov. Phys. Usp. 28, 113 (1985)].

[18] M. B. Voloshin, Once again about the role of gluonic mechanism in interaction of light Higgs boson with hadrons, Yad. Fiz. 44, 738 (1986) [Sov. J. Nucl. Phys. 44, 478 (1986)].
[19] S. Raby and G. B. West, The branching ratio for a light Higgs to decay into $\mu^{+} \mu^{-}$Pairs, Phys. Rev. D 38, 3488 (1988).

[20] R. S. Chivukula, A. G. Cohen, H. Georgi, B. Grinstein, and A. V. Manohar, Higgs decay into goldstone bosons, Ann. Phys. (N.Y.) 192, 93 (1989).

[21] R. S. Chivukula, A. G. Cohen, H. Georgi, and A. V. Manohar, Couplings of a light Higgs boson, Phys. Lett. B 222, 258 (1989).

[22] T. N. Truong and R. S. Willey, Branching ratios for decays of light Higgs bosons, Phys. Rev. D 40, 3635 (1989).

[23] J. F. Donoghue, J. Gasser, and H. Leutwyler, The decay of a light Higgs boson, Nucl. Phys. B343, 341 (1990).

[24] M. A. Shifman, A. I. Vainshtein, and V. I. Zakharov, Remarks on Higgs boson interactions with nucleons, Phys. Lett. 78B, 443 (1978).

[25] A. I. Vainshtein, V. I. Zakharov, and M. A. Shifman, Higgs Particles, Usp. Fiz. Nauk 131, 537 (1980) [Sov. Phys. Usp. 23, 429 (1980)].

[26] D. B. Kaplan and M. B. Wise, Couplings of a light dilaton and violations of the equivalence principle, J. High Energy Phys. 08 (2000) 037.

[27] M. B. Voloshin and V.I. Zakharov, Measuring QCD Anomalies in Hadronic Transitions Between Quarkonium States, Phys. Rev. Lett. 45, 688 (1980).

[28] S. Weinberg, The Quantum Theory of Fields: Modern applications (Cambridge University Press, Cambridge, England, 2013), Vol. 2.

[29] S. Scherer, Introduction to chiral perturbation theory, Adv. Nucl. Phys. 27, 277 (2003).

[30] S. Scherer and M. R. Schindler, A primer for chiral perturbation theory, Lect. Notes Phys. 830, 1 (2012).

[31] C. Roiesnel and T. N. Truong, Resolution of the $\eta \rightarrow 3 \pi$ Problem, Nucl. Phys. B187, 293 (1981).

[32] J. Gasser and H. Leutwyler, Low-energy expansion of meson form-factors, Nucl. Phys. B250, 517 (1985).

[33] G. Colangelo, J. Gasser, and H. Leutwyler, $\pi \pi$ scattering, Nucl. Phys. B603, 125 (2001).

[34] V. Guelpers, G. von Hippel, and H. Wittig, The scalar radius of the pion from Lattice QCD in the continuum limit, Eur. Phys. J. A 51, 158 (2015).

[35] J. D. Bjorken and S. D. Drell, Relativistic Quantum Fields (McGraw-Hill, New York, 1965).

[36] G. Barton, Introduction to Dispersion Techniques in Field Theory (Benjamin, New York, 1965).

[37] F. J. Yndurain, Low-energy pion physics, arXiv:hep-ph/ 0212282.

[38] M. Tanabashiet al., Review of particle physics, Phys. Rev. D 98, 030001 (2018).

[39] B. Hyams et al., $\pi \pi$ Phase shift analysis from $600-\mathrm{MeV}$ to 1900-MeV, Nucl. Phys. B64, 134 (1973).

[40] K. L. Au, D. Morgan, and M. R. Pennington, Meson dynamics beyond the Quark model: A study of final state interactions, Phys. Rev. D 35, 1633 (1987).

[41] C. Itzykson and J. B. Zuber, Quantum Field Theory, International Series in Pure and Applied Physics (McGraw-Hill, New York, 1980).

[42] R. Omnes, On the solution of certain singular integral equations of quantum field theory, Nuovo Cimento 8, 316 (1958). 
[43] N. Muskhelishvili, Singular Integral Equations: Boundary Problems of Function Theory and their Application to Mathematical Physics, 2nd ed. (Noordhof, Groningen, 1953).

[44] B. Moussallam, N(f) dependence of the quark condensate from a chiral sum rule, Eur. Phys. J. C 14, 111 (2000).

[45] F. J. Yndurain, The Quadratic scalar radius of the pion and the mixed pi-k radius, Phys. Lett. B 578, 99 (2004); Erratum 586, 439(E) (2004).

[46] B. Ananthanarayan, I. Caprini, G. Colangelo, J. Gasser, and H. Leutwyler, Scalar form-factors of light mesons, Phys. Lett. B 602, 218 (2004).

[47] F. J. Yndurain, The Scalar radius of the pion, Phys. Lett. B 612, 245 (2005).

[48] J. A. Oller and L. Roca, Scalar radius of the pion and zeros in the form factor, Phys. Lett. B 651, 139 (2007).

[49] D. H. Cohen, D. S. Ayres, R. Diebold, S. L. Kramer, A. J. Pawlicki, and A. B. Wicklund, Amplitude analysis of the $K-K+$ system produced in the reactions pi- $\mathrm{p}-\rightarrow \mathrm{K}-\mathrm{K}+\mathrm{n}$ and $\mathrm{pi}+\mathrm{n}-\rightarrow \mathrm{K}-\mathrm{K}+\mathrm{p}$ at 6-GeV/c, Phys. Rev. D 22, 2595 (1980).

[50] R. Kaminski, L. Lesniak, and K. Rybicki, New solutions for scalar-isoscalar pi pi phase shifts, AIP Conf. Proc. 432, 397 (1998).

[51] F. Guerrero and J. A. Oller, $K \bar{K}$ scattering amplitude to one loop in chiral perturbation theory, its unitarization and pion form-factors, Nucl. Phys. B537, 459 (1999); Erratum B602, 641(E) (2001).

[52] I. Caprini, G. Colangelo, and H. Leutwyler, Theoretical aspects of the pion-pion interaction, Int. J. Mod. Phys. A 21, 954 (2006).

[53] F. J. Yndurain, Some comments on calculations of the scalar radius of the pion and the chiral constant anti-1(4), arXiv: hep-ph/0510317.
[54] R. Barbieri and G. Curci, On the Higgs coupling to nucleons and pions, Phys. Lett. B 219, 503 (1989).

[55] R. Kaminski, L. Lesniak, and B. Loiseau, Scalar mesons and multichannel amplitudes, Eur. Phys. J. C 9, 141 (1999).

[56] Particle Data Group, Mesons, light unflavored, 2018, http://pdglive.lbl.gov/ParticleGroup.action?init=0\&node= MXXX005.

[57] H. Albrecht et al., Branching ratio and mass spectrum of the decay upsilon-prime $\rightarrow$ upsilon pi+ pi-, Phys. Lett. 134B, 137 (1984).

[58] J. Z. Bai et al. (BES Collaboration), psi(2S) $-\rightarrow$ pi+ pi- J/psi decay distributions, Phys. Rev. D 62, 032002 (2000).

[59] T. Himel, Decays of the psi-prime (3684) to other charmonium states. Ph.D. thesis, SLAC, 1979, http://www-public .slac.stanford.edu/sciDoc/docMeta.aspx?slacPubNumber= slac-r-223.html.

[60] M. B. Voloshin, Adler's selfconsistency condition in the decay psi-prime (3700) $\rightarrow$ psi (3100) pi pi, Pis'ma Zh. Eksp. Teor. Fiz. 21, 733 (1975); [JETP Lett. 21, 347 (1975)].

[61] M. B. Voloshin, Two-pion transitions in quarkonium revisited, Phys. Rev. D 74, 054022 (2006).

[62] M. B. Voloshin, Charmonium, Prog. Part. Nucl. Phys. 61, 455 (2008).

[63] M. A. Shifman, An estimate of the gluonium mass from QCD low-energy theorems, Z. Phys. C 9, 347 (1981).

[64] M. A. Shifman, A. I. Vainshtein, and V. I. Zakharov, QCD and resonance physics: Applications, Nucl. Phys. B147, 448 (1979).

[65] V. A. Novikov, M. A. Shifman, A. I. Vainshtein, and V. I. Zakharov, In a search for scalar gluonium, Nucl. Phys. B165, 67 (1980). 